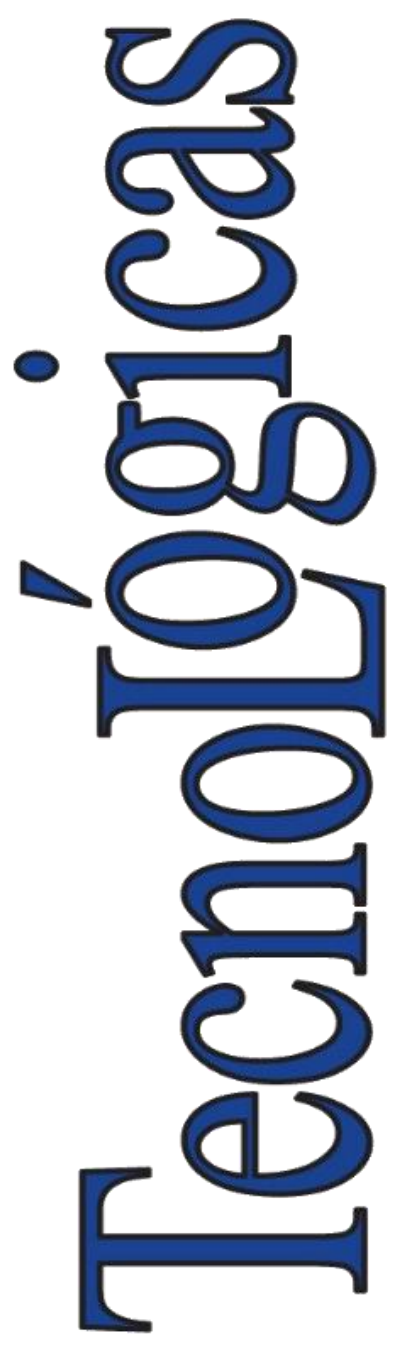

ISSN-p: 0123-7799 ISSN-e: $2256-5337$

Vol. 24, nro. 51, e1856, 2021

Recibido: 22 enero 2021 Aceptado: 12 mayo 2021 Disponible: 11 junio 2021

CInstituto Tecnológico Metropolitano

Este trabajo está licenciado bajo una Licencia Internacional

Creative Commons Atribución (CC BY-NC-SA)

\section{Comportamiento termo fluidodinámico del acero en un molde de colada continua: una revisión}

\section{Thermo-Fluid Dynamic Behavior of Steel in a Continuous Casting Mold: a review}

(D) Yordy González-Rondón'1; (D) José Eduardo Rengel-Hernández²

${ }^{1}$ Universidad de Oriente Barcelona, Estado Anzoátegui - Venezuela, yordygonzalez@hotmail.com

2 Universidad de Oriente Barcelona, Estado Anzoátegui - Venezuela, rengel66@gmail.com

Cómo citar / How to cite

Y. González-Rondón; J. E. Rengel-Hernández, "Comportamiento termo fluidodinámico del acero en un molde de colada continua: una revisión", TecnoLógicas, vol. 24, nro. 51, e1856, 2021. https://doi.org/10.22430/22565337.1856 


\section{Resumen}

Se ha realizado una revisión de la literatura para identificar qué se sabe en relación con los mecanismos de transferencia de calor, comportamiento termofluidodinámico, características de la solidificación, factores que influyen en el origen de defectos en el acero y uso de estrategias que impactan en una reducción de los defectos que se originan, principalmente, en el molde de la colada continua de acero. La metodología consistió en colectar y sintetizar conocimientos fragmentados, comparar la información encontrada en diferentes fuentes, y dar una respuesta, clara y actualizada, sobre el comportamiento termofluidodinámico del acero en el molde de colada. Como resultado de esta revisión se puede concluir que los defectos graves, como grietas y depresiones, están relacionados con el comportamiento termomecánico; las grietas se asocian al flujo turbulento, variación en el nivel del menisco, alta velocidad de colada y comportamiento inadecuado del polvo colador y la segregación se relaciona con la contracción del acero, temperatura y velocidad de colada y el flujo de calor en el contorno de la pieza. También se ha encontrado que, a pesar de la complejidad de los fenómenos que ocurren en el molde, se puede lograr la formación de una costra de acero adecuada y reducir la aparición de defectos, realizando las acciones que propicien un ajuste adecuado de los parámetros del molde. Además, es imprescindible aplicar prácticas de conicidad y oscilación del molde, configuración de buza y aplicación de campos electromagnéticos, para producir un acero de calidad.

\section{Palabras clave}

Molde de colada continua, Acero industrial, Solidificación del acero, termofluidodinámica, transferencia de calor.

\section{Abstract}

A review of the literature has been carried out to identify what is known in relation to heat transfer mechanisms, thermo-fluid dynamic behavior, solidification characteristics, factors that influence the origin of defects in steel and the use of strategies that impact on a reduction of the defects that originate, mainly, in the mold of the continuous casting of steel. The methodology consisted of collecting and synthesizing fragmented knowledge, comparing the information found in different sources, and giving a clear and up-to-date answer on the thermo-fluid dynamic behavior of the steel in the casting mold. As a result of this review, it can be concluded that serious defects, such as cracks and depressions, are related to thermomechanical behavior; cracks are associated with turbulent flow, variation in the level of the meniscus, high casting speed and inadequate behavior of the strainer powder, and segregation is related to steel contraction, casting temperature and speed, and heat flow in the contour of the piece. It has also been found that, despite the complexity of the phenomena that occur in the mold, it is possible to achieve the formation of a suitable steel crust and reduce the appearance of defects, performing the actions that promote an adequate adjustment of the parameters of the mold. Furthermore, it is essential to apply mold taper and oscillation practices, nozzle configuration and application of electromagnetic fields, to produce quality steel.

\section{Keywords}

Continuous casting mold, industrial steel, solidification of steel, thermo-fluid dynamics, heat transfer. 


\section{INTRODUCCIÓN}

El primer paso para lograr la transformación masiva del arrabio en acero, lo dio el ingeniero inglés Henry Bessemer en 1855, cuando patentó un proceso que marcó el paso decisivo en la elaboración del acero a partir del hierro producido en el alto horno en cantidades industriales y a bajo costo [1]-[3]. Más tarde en Europa, en la década de 1950, en un intento por incrementar la productividad de acero, se desarrolló el proceso de la colada continua (PCC) [4]. Se denominó continua porque permitió la solidificación del metal líquido y el retiro inmediato de un molde [5], [6]. La ventaja más importante de este proceso es la regularidad [7], y ha permitido conseguir los mayores avances en la producción de acero [8], [9], aumentando significativamente su consumo en las últimas décadas en todo el mundo [10].

En la Figura 1 se muestra que el PCC inicia cuando la cuchara recibe el acero líquido proveniente de un horno de arco eléctrico o bien del convertidor [11], allí se almacena y luego se introduce en la artesa con la finalidad de ser homogenizado [3]. Posteriormente se deja fluir a través de una boquilla de entrada sumergida (BES) hacia un molde de cobre de alta pureza de $10 \mathrm{~mm}$ a $20 \mathrm{~mm}$ de espesor [12], con longitud de $500 \mathrm{~mm}$ a $800 \mathrm{~mm}$ [11], con caras internas recubiertas de níquel o cromo para aumentar la dureza y evitar que se adhiera cobre en la superficie del acero solidificado [13], las caras pulidas a espejo (para disminuir la fricción), el molde es sin fondo y enfriado por agua [11], [14]. El acero comienza a solidificarse por el contacto con el molde refrigerado, formando una costra que durante su evolución se contrae y crea intermitencias al entrar en contacto con el molde, aun cuando este pudiera presentar una conicidad especialmente diseñado para evitarlo [1]. Para procurar que la costra no se adhiera al molde, se utilizan movimientos oscilatorios en él (mecánica o hidráulicamente) y aceites o polvos de fundición que se introducen por la parte superior del molde [11], [12]. El agregado de los polvos se realiza manualmente por operadores o por un dispositivo mecánico, y en su estadía en el molde tienden a sintetizarse y fundirse en la parte superior, formando una piscina de escoria que, con el avance del fundido de acero, se infiltra entre las paredes del molde y la costra de metal que ha solidificado. En la filtración, el polvo forma una película de escoria líquida y una capa de escoria sólida de $0.1 \mathrm{~mm}$ y $2 \mathrm{~mm}$ de espesor, respectivamente. Estos espesores influyen en el nivel de lubricación y en el flujo de calor extraído horizontalmente entre la costra y el molde [15], [16].

El proceso continúa con la salida del acero por la parte inferior del molde (llamado hilo), conteniendo metal líquido en su interior [11] y una costra de acero solidificada en el exterior, las cuales, de ser muy pequeñas, pueden producirse desgarros y roturas, y, de ser demasiado gruesas, pueden generar problemas en el curvado posterior al molde [17]. Por estas razones, conviene que la costra alcance un espesor de $12 \mathrm{~mm}$ a $25 \mathrm{~mm}$, lo suficiente para resistir la presión ferrostática que ejerce el acero líquido sobre ella [8], [18]-[20]. El hilo continúa en la zona de enfriamiento secundario, donde se le aplica agua pulverizada y aire con la finalidad de culminar la solidificación iniciada en el molde (completando la longitud metalúrgica [21]), y el proceso finaliza cuando el producto colado es cortado a la longitud deseada en forma de planchones, palanquillas o tochos por medio de sistemas oxicortes [1], [6], [18], [22]. Durante la solidificación, el $15 \%$ del calor se extrae en el molde, entre el $30 \%$ y $40 \%$ en la zona de enfriamiento secundario, un $10 \%$ en otras partes del PCC, y el resto a la atmósfera. 


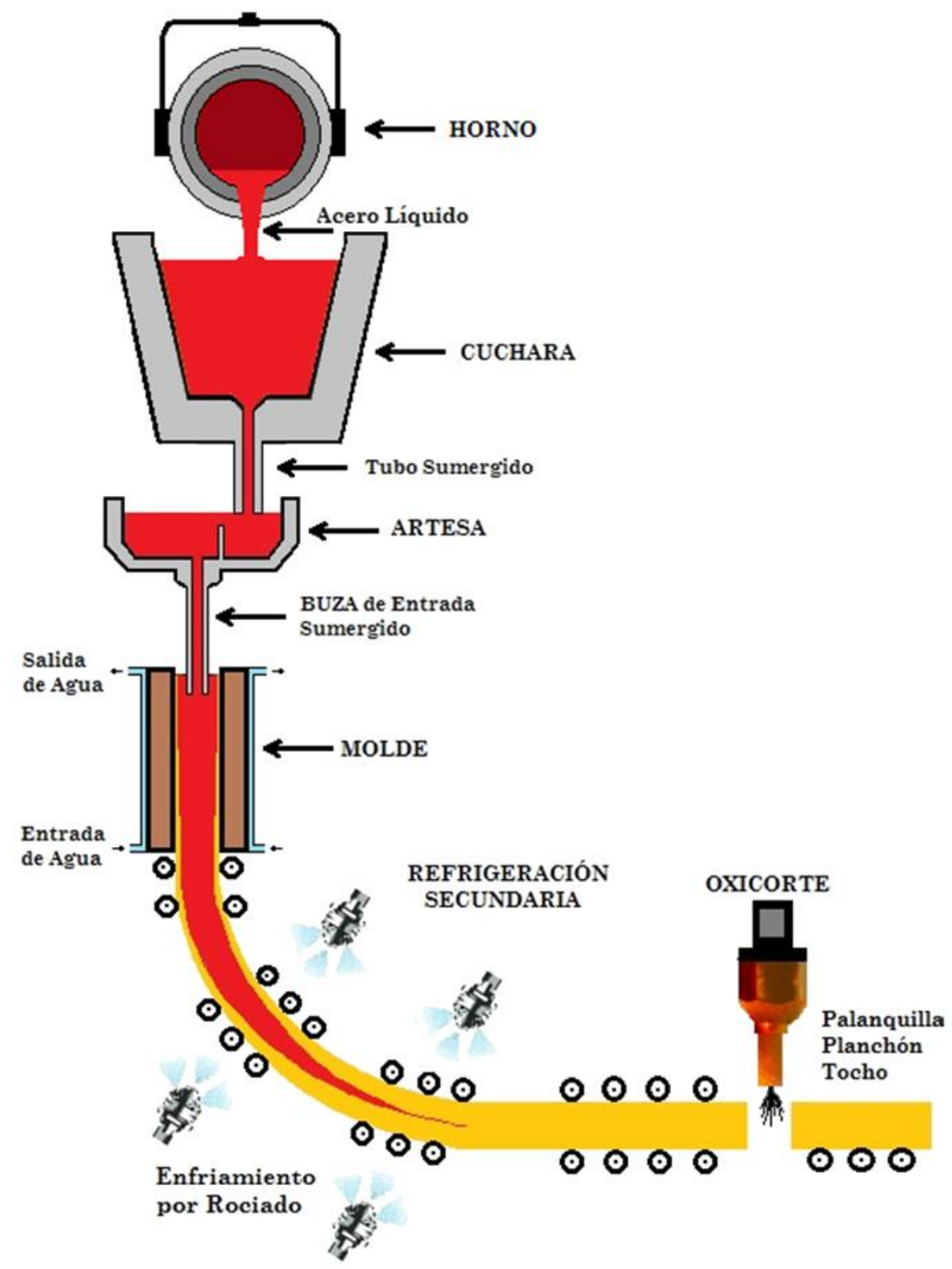

Figura 1. Proceso de la colada continua del acero. Fuente: elaboración propia.

El objetivo principal del PCC es extraerle calor al acero líquido en un tiempo muy corto [1], [16]; y de todos los elementos que conforman el PCC, el molde es el más importante [1], [11], [23] debido a que en él se debe crear una costra sólida de acero con características específicas y, al mismo tiempo, se deben controlar los fenómenos de transferencia de calor, transformación de fase y flujo de fluidos [22], [24], [25]. La concurrencia de estos fenómenos y ciertas condiciones de funcionamiento complican el proceso en el molde [23]. Por ejemplo, si aumenta la velocidad de colada se afecta la infiltración de escoria y ésta, a la vez, condiciona la lubricación y la transferencia de calor entre la costra sólida y el molde, además aumenta la turbulencia en el flujo de acero líquido y podría causar una distorsión en la región donde el flujo de acero impacta con la costra solidificada [15]. Aunado a éstos, durante la solidificación el acero adquiere la mayoría de los defectos en los productos colados, tales como marcas, segregación, grietas, inclusiones, entre otros [9],[26]-[29], quienes influyen en las propiedades 
mecánicas; y para disminuir estos defectos, se deben ajustar los parámetros operacionales [7], [9] venciendo las principales dificultades que son: adoptar una velocidad de colada a la velocidad de solidificación y establecer las condiciones de enfriamiento y lubricación en el molde [1].

La mayoría de los defectos que se forman durante la solidificación del acero mediante el PCC son muy difíciles de corregir en etapas posteriores. Esta es una de las razones de mayor peso que ha hecho que la comunidad científica se interese en determinar lo que ocurre en la solidificación del acero, para así poder incrementar el rendimiento de las instalaciones sin sacrificar la calidad, cumpliendo de este modo con las rigurosas normas [30]. Aunque el PCC parece simple, implica una gran complejidad metalúrgica, por lo cual es necesario comprenderlo y evaluarlo para optimizar las instalaciones y mejorar los productos [5].

Todavía hay muchos desafíos que superar en este campo de investigación [31], por lo cual este trabajo surge como una revisión amplia de investigaciones que tratan los fenómenos que ocurren en el molde de colada continua de acero. Entre estos se destacan los mecanismos de transferencia de calor que ocurren en el molde, la influencia de la temperatura de colada y solidificación, la velocidad de colada y enfriamiento del acero, la influencia de los elementos aleantes y el contenido de carbono en la solidificación del acero, el fenómeno de la contracción del acero, la formación de segregado, el uso de aceites y polvos coladores, la inclusión de buzas sumergidas, la estrategia de aplicar agitación magnética y la implementación de los osciladores en el molde.

\section{METOdOLOGía}

El contenido que se presenta en este artículo surgió de una revisión bibliográfica relevante sobre el comportamiento termofluidodinámico que experimenta el acero en el molde de un proceso de colada continua, con la finalidad de identificar qué se sabe y qué se desconoce del tema. Se trató de una revisión descriptiva de la literatura, donde se identificaron los correctos procedimientos para redactar un artículo de revisión, y se dejó a la vista la constante evolución del tema de estudio [32]. Esta revisión es de gran utilidad e interés en la enseñanza, y campos conexos, que estudian lo relacionado a los factores que influyen el origen de los defectos en el acero, las características que intervienen durante la solidificación de este, los mecanismos de transferencia de calor, el comportamiento termofluidodinámico en un molde de colada y el uso de estrategias para la reducción de defectos en el acero, entre otros. También se cumplen con las funciones de compactar y sintetizar conocimientos fragmentados sobre el comportamiento del acero en el molde de colada, trasmitir al lector nuevos conocimientos reportados sobre los fenómenos que se presentan en el molde, comparar la información encontrada en diferentes fuentes, conocer la estrategia de los investigadores para minimizar los defectos que se originan en el molde y contribuir a la docencia [33], [34].

Luego de la definición del tema y de la elaboración de un plan de trabajo, la búsqueda bibliográfica consistió en obtener tesis, textos y publicaciones de artículos durante los últimos años, sin excluir publicaciones más antiguas, pero que, por su aporte a la ciencia, aún son de interés para el desarrollo de este trabajo. Se utilizaron los buscadores académicos Scopus, Google Académico, Academia.edu, ScienceResearch.com, Springer Link, así como la visita a diferentes páginas web de revistas especializadas en el área de estudio. La metodología de búsqueda fue iterativa [35] por la interrelación que guardan los aspectos en el molde, y la sistematización de la información resultante originó la búsqueda de nueva información por la necesidad de profundizar en el tema. La información analizada de una o más fuentes fue sometida a un proceso de condensación y destilación, seleccionando las partes más 
sustanciales de cada trabajo revisado [36]. Finalmente se procedió a preparar un guion para la construcción del artículo de revisión con la finalidad de servir de guía en la redacción, organización y mantener un orden lógico.

\section{RESULTADOS Y DISCUSIÓN}

\subsection{Transferencia de calor en el molde}

El molde de la colada continua de acero experimenta cambios térmicos que influyen en la calidad de las piezas fundidas [37]. La extracción de calor se debe, principalmente, al agua de enfriamiento que circula por las paredes del molde [12], [38]. El máximo flujo de calor que se extrae corresponde al sitio donde inicia la solidificación, es decir, en el menisco, porque es allí donde el acero líquido está en contacto con el molde de cobre [16]. A medida que progresa la solidificación, una costra de acero solidificado se va formando y posteriormente separando de la pared del molde, creando una brecha (producto de la contracción del acero y de la deformación del molde) que causa reducción en el flujo de calor extraído [6], [12], [39].

La Figura 2 muestra cómo se transmite el flujo de calor desde el acero líquido hasta el agua de enfriamiento que circula por las paredes del molde [16], [40], siendo la conducción el mecanismo de transferencia de calor dominante en el sistema [20], [41].

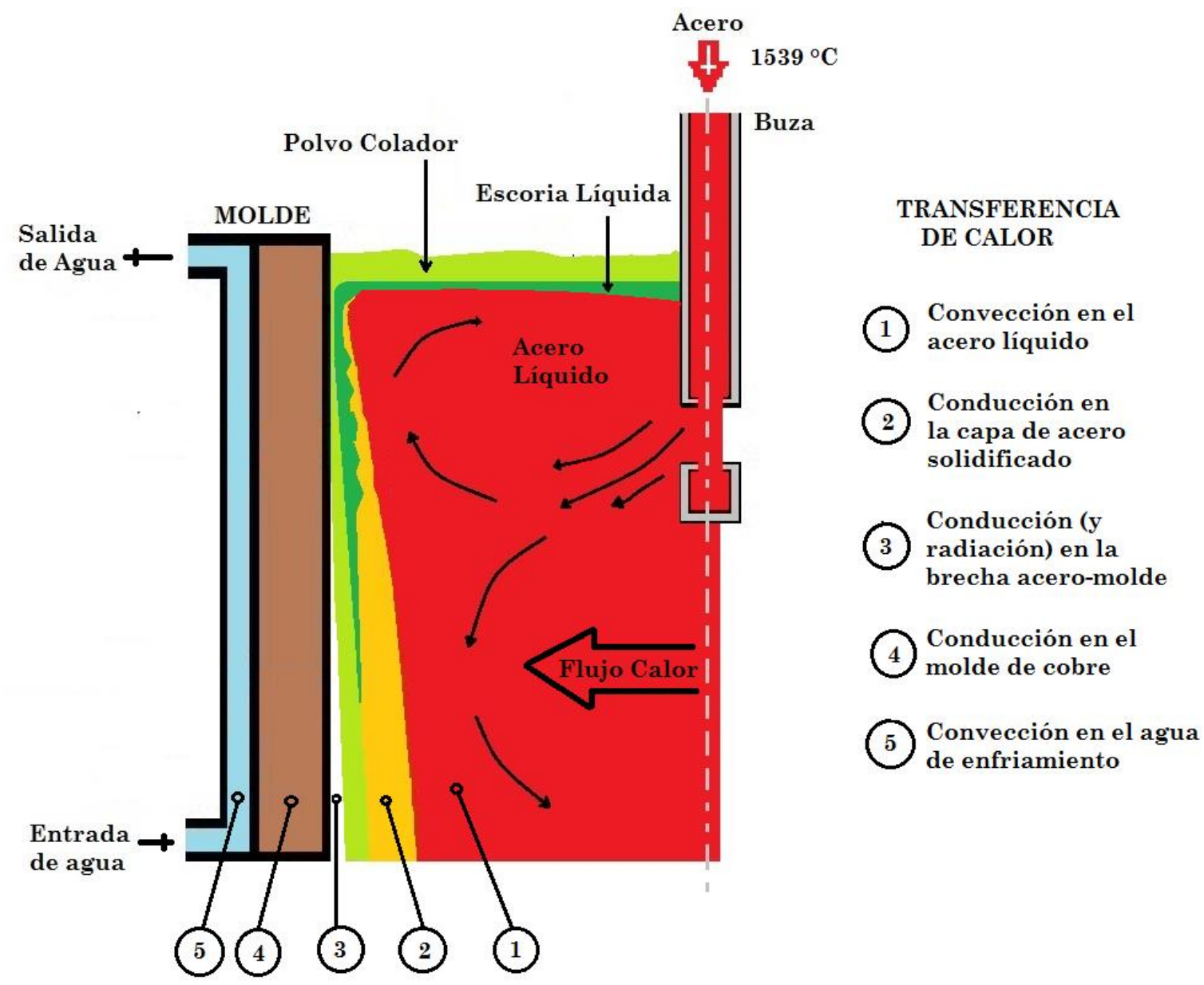

Figura 2. Transferencia de calor en el molde. Fuente: elaboración propia. 
El molde puede analizarse como una pared de múltiples capas que transfiere el calor desde el acero fundido al agua de enfriamiento. Asumiendo un estado estable y un flujo de calor unidimensional en la pieza colada, por simplicidad, el flujo de calor desde el acero líquido al agua de enfriamiento puede representarse por (1) [12], [42], [43], [44]:

$$
\dot{q}=h\left(T_{l}-T_{w}\right)
$$

Donde, $\dot{q}$ es el flujo de calor, $h$ es el coeficiente global de transferencia de calor entre el acero líquido y el refrigerante, $T_{l}$ la temperatura del acero líquido y $T_{w}$ la temperatura del agua de enfriamiento. Pero $h$ puede definirse por una serie de resistencias térmicas encontradas al flujo de calor, como se observa en (2) [12],[38],[42],[44]:

$$
\frac{1}{h}=\frac{1}{h_{1}}+\frac{X_{2}}{K_{X_{2}}}+R_{g}+\frac{X_{4}}{K_{X_{4}}}+\frac{1}{h_{5}}
$$

Siendo $h_{1}$ el coeficiente de transferencia de calor por convección en el acero líquido, $X_{2}$ el espesor del acero sólido, $K_{X_{2}}$ la conductividad térmica del acero sólido, $R_{g}$ la resistencia por conducción (y radiación) a través de la brecha de acero - molde, $X_{4}$ el espesor del molde, $K_{X_{4}}$ la conductividad térmica del molde y $h_{5}$ el coeficiente de transferencia de calor por convección en el agua de refrigeración. Partiendo del supuesto caso que el coeficiente $h$ en términos porcentual equivale al $100 \%$ de la resistencia encontrada al flujo, las porciones que le corresponde a cada resistencia térmica (descritas en la Figura 2) variarán de acuerdo con la posición que se esté estudiando en el molde, y esto se debe a que el acero durante su recorrido en el molde experimenta cambios constantemente. Cualitativamente, las resistencias, debido a la pared del molde y al agua de enfriamiento, son las que tienen menor incidencia [12], mientras que la resistencia térmica que corresponde a $R_{g}$ representa la de mayor incidencia [21] según la ubicación. En [45] se realizó una investigación sobre el equilibrio térmico del molde de la colada continua de acero. La metodología consistió en fijar el espesor de la costra de acero solidificado en $10 \mathrm{~mm}$ y el espesor de la pared del molde de cobre en $20 \mathrm{~mm}$; con base en los resultados, el estudio de [16] determinó los porcentajes de resistencias térmica del molde, mostrados en la Tabla 1. Se observa que la zona acero-molde es la que ejerció mayor influencia sobre la transferencia de calor, seguida por el espesor de la costra de acero solidificado, y la que menos influyo fue la zona molde-agua. Es importante resaltar que los resultados de la Tabla 1 corresponden a algún punto comprendido entre el menisco y la parte inferior del molde, y de hacer un análisis en cualquier otro punto del molde, las resistencias térmicas cambiaran y el flujo de calor extraído se modificara. Esto se debe a que el espesor del acero solidificado, los espesores de las capas que derivan del polvo colador y la brecha de aire que se forma entre el acero -solidificado-molde cambian en el sentido de la colada [16], [21], [43]. 
Tabla 1. Aporte en la transferencia de calor en un molde de colada continua de acero. Fuente: [16].

\begin{tabular}{lccccc}
\hline Características & $\begin{array}{c}\text { Acero líquido- } \\
\text { sólido }\end{array}$ & $\begin{array}{c}\text { Acero } \\
\text { sólido }\end{array}$ & $\begin{array}{c}\text { Brecha acero- } \\
\text { molde }\end{array}$ & Molde & Molde-agua \\
\hline $\begin{array}{l}\text { Coeficiente de } \\
\text { transmisión }\left(\mathrm{kW} / \mathrm{m}^{2} \mathrm{~K}\right)\end{array}$ & 10 & 3 & 2 & 20 & 40 \\
\cline { 1 - 1 } & 10 & 33 & 50 & 5 & 2 \\
\hline
\end{tabular}

La conductividad térmica del acero depende de la composición química. Y para tener una valoración del flujo de calor que se extrae en el molde desde el acero líquido al agua de enfriamiento, deben conocerse con precisión los valores de coeficientes de transferencia de calor de las zonas descritas en la Figura 2. Tal requerimiento no es fácil de conseguir por la complejidad del sistema y sobre todo por la dinámica que ocurre entre en la zona acero-molde [21]. En la zona de convección del acero líquido, una simplificación importante es la propuesta por [46] en sustituir el efecto de la convección por una conductividad térmica efectiva. En [47] demostraron que los altos valores en la conductividad térmica efectiva del acero líquido disminuyen el espesor de la capa solidificada, incrementan la temperatura de la superficie del hilo en la parte superior y acortan la longitud metalúrgica, llegando a la conclusión que en la zona líquida la conductividad influye en la disipación del recalentamiento. En el trabajo de [16] se mostró la expresión (3), que trata al acero líquido como un sólido con una conductividad térmica efectiva $k_{L}^{e f}$ varias veces superior al valor real según el factor $\beta_{e f}$ :

$$
k_{L}^{e f}=\beta_{e f} k_{S}
$$

Donde $k_{S}$ es la conductividad del acero a la temperatura solidus y $\beta_{\text {ef }}$ el factor de incremento del acero líquido. En el estudio de [47] aplicaron la expresión (4) utilizando diferentes enfoques para calcular la conductividad térmica del acero en estado líquido, líquido-sólido (zona pastosa) y sólido.

$$
k_{e f f}=M_{e f f} \cdot k\left(T_{s}\right)
$$

Siendo $k_{e f f}$ la conductividad térmica efectiva del líquido, $M_{e f f}$ la relación entre la conductividad efectiva y la conductividad real del acero líquido, y $T_{s}$ la temperatura del acero sólido. En [43] se mostró un modelo lineal y otro cuadrático para determinar la conductividad térmica del acero en estado líquido, pastoso y sólido en el molde. En la zona de conducción en el acero sólido, la conductividad térmica del acero sólido se ha asumido constante [48], [49] o lineal con la temperatura [50], sin embargo, no debe descuidarse su influencia, porque habrá una mayor transferencia de calor donde la capa de acero es más delgada (en el menisco) y disminuirá con la longitud del molde. Además, por tratarse de una aleación, se formará una zona pastosa cuya conductividad térmica dependerá de las fases [11], [12], [16], y esto afecta la transferencia de calor. En la zona de conducción (y radiación) acero-molde, es prácticamente imposible obtener un coeficiente de transferencia con cierto grado de precisión [18] debido: a) la formación de hueco de aire o brecha entre el molde y el acero, de ancho variable no uniforme, producido por la contracción del acero, b) la incorporación del espesor del lubricante de colada en las distintas capas que se crean (líquida, cristalina y vítrea), c) la transferencia de calor radiactivo a través de la brecha, d) al efecto de la velocidad de fundición sobre los parámetros antes mencionados y el sobrecalentamiento, entre otros. Estas razones, 
han llevado a utilizar un coeficiente de transferencia de calor promedio en los modelos matemáticos obviando que el coeficiente cambia a lo largo de la sección del molde [12], [18], así como el uso de coeficientes distintos para ciertas posiciones en el molde [11], [16], o un coeficiente de transferencia de calor medido experimentalmente [51]. En la zona de la pared del molde, la conducción de calor es gobernada principalmente por la conductividad térmica del material, que normalmente es cobre o alguna aleación de este metal [12]. Luego de muchos ciclos de calentamiento y enfriamiento, el molde experimenta deformaciones residuales permanentes (desgaste y distorsión), por lo que se procede a maquinar, hasta que las paredes se reduzcan a un grosor mínimo permitido [37]. La distorsión térmica conduce a grietas por fatiga y compromete la geometría interna del molde, lo que afecta la transferencia de calor en la costra solidificada [21], [37]. Con la aplicación de moldes ranurados (mayor resistencia térmica) y el uso de materiales de menor conductividad térmica, se ha logrado reducir el flujo de calor en la zona del menisco, mejorando la calidad de los productos, principalmente en aceros con aproximadamente $0.1 \%$ de carbono [16], [52]. En la zona de convección en el agua de enfriamiento, la circulación del refrigerante se hace en el mismo sentido de avance de la pieza de colada o, al contrario. La transferencia de calor se puede estimar empleando la expresión de Dittus-Boelter [16], mostrada en (5), si el régimen de flujo en los canales es turbulento:

$$
h_{M A}=0,023 \frac{k_{A}}{L_{A}} \operatorname{Re}_{a}^{4 / 5} \operatorname{Pr}_{a}^{0,3}
$$

Donde $k_{A}$ es la conductividad térmica del agua, $L_{A}$ longitud del canal de refrigeración, $R e_{a}$ número de Reynolds del agua de refrigeración y $P r_{a}$ el número de Prandtl del agua de refrigeración. La ecuación (5) puede emplearse cuando no se producen núcleos de ebullición en los canales, de manera que es posible que su aplicación pueda originar errores considerables [16] sino se cumple la condición en el proceso. En [45] se informó que una velocidad del agua de $6 \mathrm{~m} / \mathrm{s}$ proporciona suficiente intercambio de convección forzada para evitar cualquier riesgo de formación de vapor y aumento de la resistencia térmica. La pieza colada puede tomar la forma de romboidicidad, lo que se ha asociado con la ebullición asincrónica en el canal de agua de refrigeración, siendo la región más afectada el área cercana al menisco. Esto se debe a que la baja velocidad del agua produce una ebullición asincrónica, intermitente en las cuatro caras frías del molde, lo que hace que se enfríen a velocidades desiguales provocando una contracción no uniforme en la costra sólida y con forma de rombo, ya que las caras más frías se contraen más que las caras más calientes [53], [54]. Actualmente, estos defectos de romboidicidad y grietas pueden ser mitigados colando a alta velocidad, gracias al surgimiento de nuevos diseños de molde denominados comercialmente Convex, Diamold y Wave [52].

Obtener una expresión que considere la mayoría de las características que se presentan en los mecanismos de transferencia de calor de la Figura 2 no es algo sencillo. Sin embargo, es posible determinar el flujo de calor a lo largo del molde, relacionando la distribución longitudinal del flujo y el calor total extraído por medio de (6), [16]:

$$
Q_{G}=\frac{1}{t_{m}} \int_{0}^{t_{m}} q(t) d t
$$


Donde $t_{m}$ es el tiempo de residencia del acero en el molde y $q$ el flujo local de calor en el molde. Bajo esta metodología, también es posible determinar un coeficiente de transferencia en la región del molde $h_{m}$ para describir la distribución del flujo de calor, como una función del tiempo de residencia del acero en el molde. Por ejemplo, en (7) el coeficiente considera una resistencia térmica debido a la formación de una brecha por el aire [25]:

$$
h_{m}=1004,6_{\text {exp }}[-0,02 t]
$$

Donde $t$ es el tiempo de permanencia del acero en el molde, que se calcula al dividir la velocidad de colada $V_{c}$ entre la altura del molde $Y$. El flujo global de calor extraído en el molde se determina al medir la variación de temperatura en el agua de enfriamiento y el caudal de este que fluye por los canales. Si se requiere obtener resultados más precisos, en cómo se distribuye el flujo de calor a lo largo del molde, puede medirse los cambios de temperaturas en distintas posiciones del molde (por medio de termopares). Luego, por medio de modelado matemático, se determina el flujo de calor que hace coincidir con los valores de temperatura medidos experimentalmente. En [16] y [43] se describen una serie de correlaciones analíticas que provienen de ensayos en molde y han sido ampliamente utilizadas en distintos trabajos como condición de contorno para calcular el enfriamiento y solidificación del acero en la zona del molde.

\subsection{Temperatura de colada y solidificación}

La temperatura de colada es la que presenta el acero a la entrada del molde, descrita como $1539{ }^{\circ} \mathrm{C}$ en la Figura 2, y tiene alta responsabilidad en la distribución de la temperatura en la zona del molde [38], [55]. Esta temperatura también influye en la producción [16], [56] y en la calidad interna de las palanquillas, cuanto más elevada es la temperatura, los rechupes y los defectos en la zona central son más severos y más fuerte la segregación axial en esa zona [17]. La importancia de esta temperatura también radica en que, si el metal está muy por debajo de ella, puede no fluir correctamente en el molde, y si está muy por encima, puede conducir a una fusión gaseosa y formar burbujas en el vaciado. En la industria es común usar una referencia aproximada de esta temperatura, y consiste en fijarla a la salida del horno de $80{ }^{\circ} \mathrm{C}$ a $150{ }^{\circ} \mathrm{C}$ superior a la de liquidus, o fijarla en la artesa (antes de colar el acero en el molde) de $20{ }^{\circ} \mathrm{C}$ a $50{ }^{\circ} \mathrm{C}$ superior a la de solidificación, y esto es conocido como sobrecalentamiento [14]. La cantidad de sobrecalentamiento del acero (que representa al calor sensible contenido en el metal líquido por encima de la temperatura liquidus [57]) no es fácil de controlar y dependerá de la temperatura de vertido del acero fuera del horno, la temperatura en la cuchara y la temperatura de la artesa [58]. El sobrecalentamiento, al igual que la velocidad de enfriamiento, influyen en gran medida en el grosor de la costra solidificada [58] y el cambio de morfología del grano, pues el grano columnar crece a medida que aumenta el sobrecalentamiento [59]. En [26] se examinó el efecto del aumento de la temperatura del acero en la artesa, y se encontró que a mayor sobrecalentamiento se redujo la incidencia de defectos en los productos finales. El sobrecalentamiento del acero también afecta la transferencia de calor en el molde: un aumento de $20^{\circ} \mathrm{C}$ de la temperatura del acero líquido incrementa de $6 \%$ a $8 \%$ el calor eliminado en el molde. Específicamente en el caso de planchones, se ha encontrado una influencia más notable del sobrecalentamiento en la extracción de calor de las caras angostas, posiblemente esto se debe al impacto de la corriente de acero líquido con el molde sobre estas caras [16]. Mediante simulaciones numéricas, en [21] se reportó como se disipa el sobrecalentamiento en el molde. Las regiones más frías se 
encontraron en el menisco, en las esquinas superiores cerca de la cara estrecha y cerca de la BES. Esto es preocupante porque podría provocar la solidificación del menisco creando un borde grueso, lo que provocaría problemas de calidad como marcas de oscilación profunda, grietas y otros defectos superficiales [21]. La temperatura a la salida del molde estuvo cercana a la temperatura liquidus [21], lo que indica que la mayor parte del sobrecalentamiento se disipa en el molde [57].

En cuanto a la temperatura de solidificación, ésta varía principalmente con el contenido de carbono. Los aceros no solidifican en un punto único, hay una brecha en la que se encuentran en estado líquido y sólido (pastoso), que se hace mayor a altos contenido de carbono [21]. Los aceros no aleados solidifican en un punto que lo fija el sitio en que la vertical correspondiente al contenido de carbono corta a la línea de líquido en el diagrama hierro carbono. En los aceros que son aleados la temperatura de solidificación se determina mediante una fórmula polinómica en la que el minuendo es $1535{ }^{\circ} \mathrm{C}$ (punto de fusión del hierro puro) y los sustraendos son una serie de funciones de monomios. Cada función expresa numéricamente la influencia del elemento de aleación respectivo. Una característica importante de los aceros aleados es que los elementos que forman la aleación hacen descender la temperatura de solidificación y por ello sus temperaturas de colada son inferiores a las de aceros ordinarios con el mismo contenido de carbono [17].

\subsection{Velocidad de colada y enfriamiento}

La velocidad de colada es la rapidez con que se vierte el metal fundido en el molde. La longitud del molde crece con la velocidad de colada, ya que de esta longitud depende el tiempo de residencia del acero en el molde y en gran medida su solidificación [1]. Un aumento en esta velocidad trae como consecuencias problemas operacionales y formación de defectos con mayor frecuencia [56], así como también mayor penetración del flujo en el molde y fluctuaciones (turbulencias) [6], [26]. En la parte superior del molde estas fluctuaciones son ocultadas por los polvos coladores, por tal razón, la dinámica del fluido en esta zona pareciera ser un proceso muy pacífico; sin embargo, esto es muy engañoso [15] porque podría generarse atrapamientos de escoria proveniente de los polvos coladores en el área del menisco [24]. El atrapamiento de escoria ocurre por un efecto de desprendimiento que se genera en la esquina superior del molde, debido a que la velocidad del flujo de acero que proviene de la cara del molde es mucho mayor que la que tiene la escoria, como se denota en la Figura 3 con el número (1). También por el mismo fenómeno puede ocurrir la formación de vórtices de Karman, representado por el número (2) en la Figura 3, causado por la separación no estacionaria de la capa de fluido al pasar sobre cuerpos de escoria sumergidos [15]. Asimismo, puede ocurrir el atrapamiento de una burbuja de argón que escapa de los puertos de la BES y alcanza la interfaz de polvo de acero-molde, mostrado con el número (3) en la Figura 3, [60].

La velocidad de colada también influye en la extracción de calor en el molde. En el estudio de [42] se observó cómo la recirculación del acero líquido en el molde produce un aumento de la transferencia de calor del acero hacia el frente de solidificación (zona de mayor velocidad y turbulencia del acero líquido), causando que se detenga el avance del frente de solidificación. 


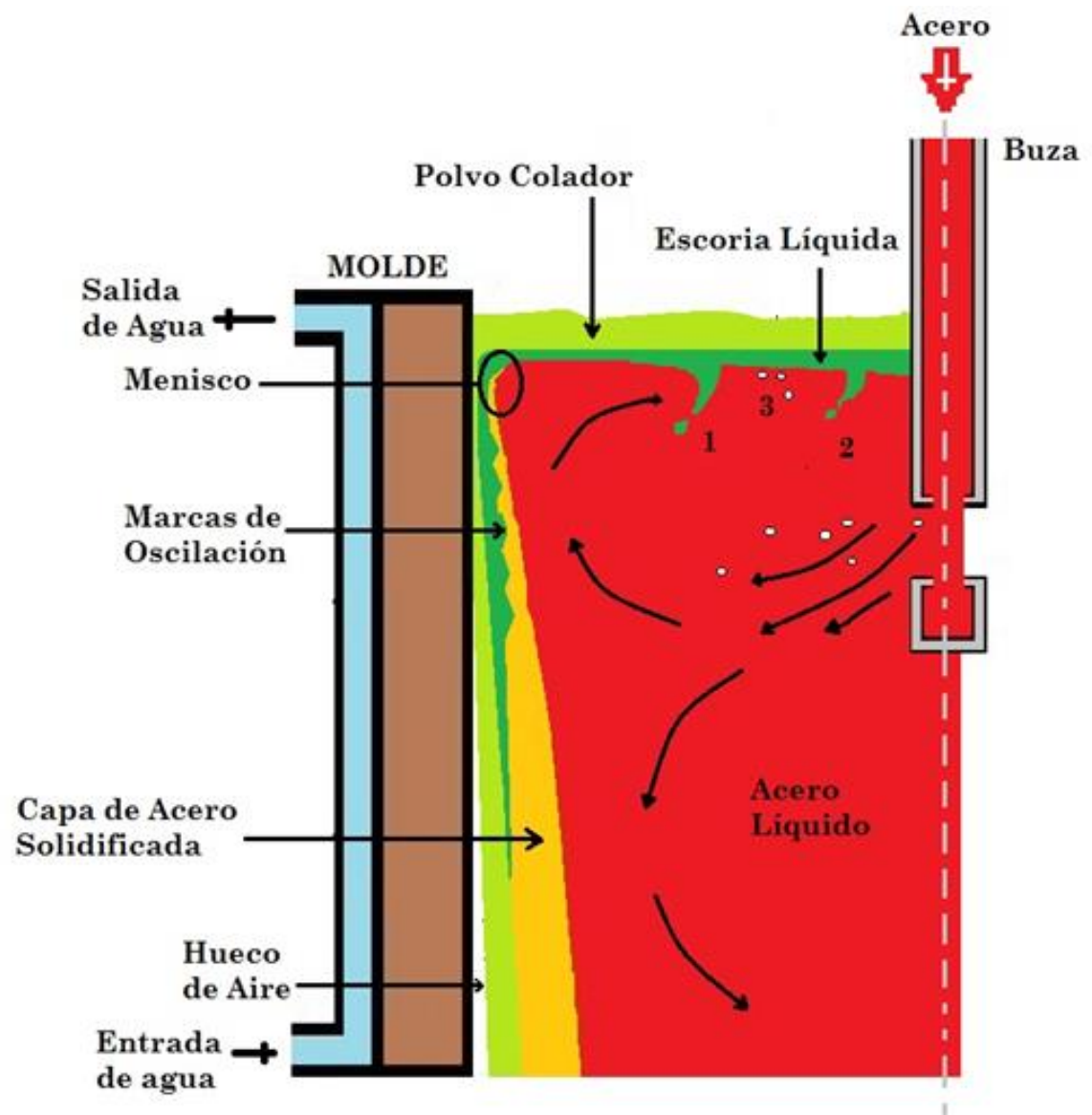

Figura 3. Esquemas de mecanismos involucrados en el atrapamiento de escoria. Fuente: elaboración propia.

Esto es perjudicial porque existe la probabilidad de rotura de los productos a la salida del molde como consecuencia de un excesivo estrechamiento de la capa solidificada. Como consecuencia de un aumento en la velocidad de colada: a) se incrementa el flujo global de calor extraído, esta dependencia se ha verificado incluso para el colado de diferentes formas geométricas, b) disminuye el tiempo de residencia del acero dentro del molde, c) disminuye la disipación del sobrecalentamiento que presentaba el acero al momento de entrar al sistema, d) disminuye entonces el espesor de la costra solidificada, y e) el proceso de solidificación comienza más tarde [16], [43].

La velocidad de enfriamiento es otro parámetro importante y está relacionada con la rapidez con que se le extrae calor al acero líquido en el molde [18]. Durante el enfriamiento, el avance del frente de solidificación se hace vulnerable, y esto se debe a que hay una estrecha relación entre la velocidad con que se realiza el enfriamiento como el intervalo de solidificación de la aleación, este fenómeno se muestra en la Figura 4. 


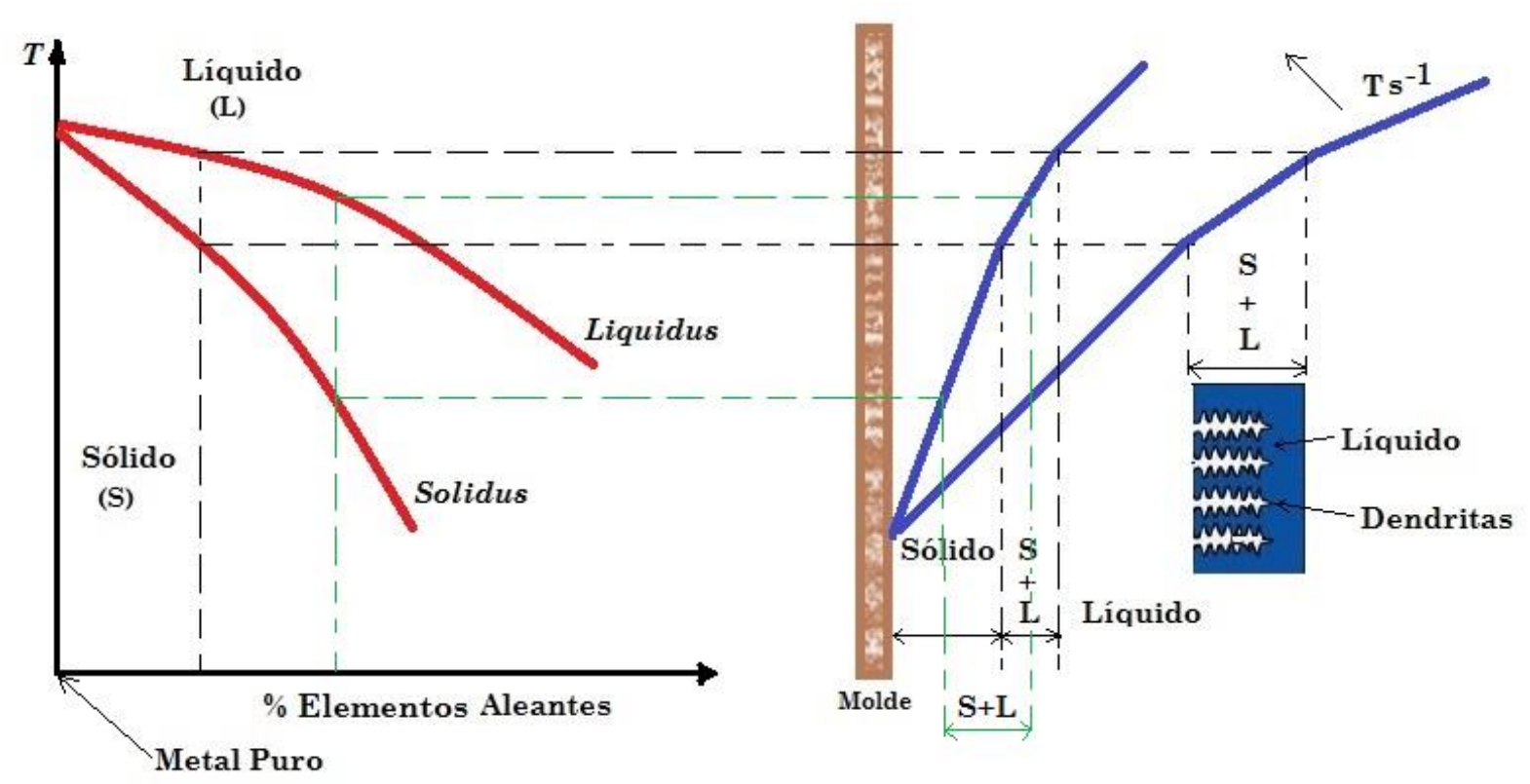

Figura 4. Intervalo de solidificación y velocidad de enfriamiento de una aleación metálica Fuente: elaboración propia.

Conforme aumenta la velocidad de enfriamiento (que corresponderá a un gradiente térmico más elevado) se hace mayor la pendiente de la curva de distribución de temperaturas, y esto causa una disminución en la extensión de la región parcialmente solidificada, pudiendo desencadenar en la formación de grietas internas [2], [18], y conducir a una microestructura anormal con falta de compactación, que tiende a disminuir la tenacidad y resistencia al impacto en los productos de fundición; y la causa raíz se debe al estrés térmico y el estrés mecánico en el proceso de enfriamiento y solidificación del acero [61]. En [62] determinaron que, a mayor velocidad de enfriamiento, la superficie del acero presenta una rugosidad suave y también explicaron que la rugosidad de la superficie del fundente se volvió rugosa para un acero con medio carbono en comparación con un acero de bajo contenido de carbono. También con un enfriamiento más intenso se destaca el aumento de las grietas longitudinales [56].

\subsection{Contenido de carbono y elementos aleantes en el acero}

Los aceros son aleaciones de hierro con pequeñas cantidades de carbono, que suelen contener otros elementos como Mn, Si, P, S, Ni, Cr, Cu, Ti [63], con la finalidad de conseguir mejoras en las propiedades mecánicas. Durante el proceso de colada continua de acero, la variación en los elementos aleantes, además de generar cambios en la composición química, afectara la conductividad térmica de la aleación. También se ha observado que los cambios en los elementos de la aleación modifican el espesor de la costra solidificada en el molde durante el proceso de colada [58], al igual que la zona pastosa (solido + liquido) [64]. Lo antes dicho, puede explicarse en la Figura 4, trazando líneas verticales en distintos porcentajes de los elementos aleantes que corten las curvas de liquidus y solidus y luego proyectando con líneas horizontales en la curva de velocidad de enfriamiento. Este comportamiento es un factor importante durante la solidificación del acero, porque conduce a una multitud de microestructuras y, por lo tanto, afectan al comportamiento del material. La estructura de solidificación también suele cambiar con el tamaño del lingote y las condiciones de la colada continua [17]. 
Cuando el porcentaje de carbono en el acero es próximo $0.1 \%$, se presenta un fenómeno interesante durante la solidificación del metal en el molde. En [16] se explicó que en instalaciones piloto como en plantas industriales se ha determinado que los aceros con esta composición presentan un mínimo en la extracción de calor en el molde, y que el incremento del flujo de calor es menor con la velocidad de colada. En [39] se hace referencia a que la velocidad de transferencia de calor para aceros de bajo carbono es menor que para el acero de medio carbono. El menor flujo de calor en el molde se explica por los espacios de aire que se forman entre la costra solidificada y el molde, producto de la contracción producida por la transformación peritéctica $(\delta+L \rightarrow \gamma)$ que sufre el material al enfriarse, y esto predomina cuando el acero presenta cercanías al $0.1 \%$ C [1], [65]. En lo que respecta a la calidad del acero, cuando los contenidos de carbono son próximos al $0.1 \%$, se crea una superficie ondulada o irregular que no se produce para mayores o menores valores porcentuales de carbono. Se ha argumentado que, para mayores porcentajes de carbono, parte del líquido pasa directamente a la fase $\gamma$ siendo menos importante el efecto de la contracción, mientras que para aceros con menores porcentajes de carbono la transformación se produce a temperaturas más bajas, superponiéndose con la contracción de origen térmico [65]. Hay otros hallazgos que se han publicado con relación a los aceros con composiciones cercanas al $0.1 \% \mathrm{C}$, en los que resaltan el origen de fisuras longitudinales y transversales y mayor tendencia a la producción de perforaciones de la línea [1], [16]. Otro elemento de interés es el aluminio. Se ha informado [66] que la variación de la composición química, la inestabilidad de la viscosidad y el deterioro de otras propiedades termofísicas en el flujo de acero, son las principales razones de ruptura, transferencia de calor desigual a través del flujo del molde, lubricación inadecuada y la mala calidad de la superficie de las losas de fundición en aceros con altos contenidos de aluminio.

\subsection{Contracción del acero}

El acero durante la solidificación en el molde se contrae, provocando un déficit de volumen que se manifiesta en forma de defectos como microporosidad y depresiones superficiales. Las depresiones son mucho más comunes en aceros con $0.1 \%$ y $0.15 \%$ en peso de $\mathrm{C}$, porque tienden a experimentar mayores fuerzas de contracción que se acumulan inmediatamente después de la solidificación inicial [66]. Durante la contracción se incrementa la formación de huecos de aire entre la costra sólida y el molde. Esta formación se describe en la Figura 5, identificados por una región de contacto completo, una región de contacto intermitente y una región de separación completa [1]. Al iniciar el fenómeno, la primera región toma en cuenta el sistema que rige el flujo de calor desde el menisco: capa solidificada, polvo en fase líquida y fase vítrea, molde y agua de enfriamiento. Enseguida se presenta una zona de intermitencia en la que el flujo de calor cambia por la formación de huecos llenados ya sea por aire o por escoria (polvo) en fase vítrea. Esta forma de intermitencia ocurre debido al gradiente de temperatura en la primera región, causando una contracción abrupta de la capa solidificada y separándola del molde [1], [67]. Al no estar la costra en contacto con el molde, la capa sufre un recalentamiento (por el calor transportado desde el centro del molde a través del metal líquido) y se vuelve a expandir haciendo contacto nuevamente con el molde, lo que explica la intermitencia [1]. 


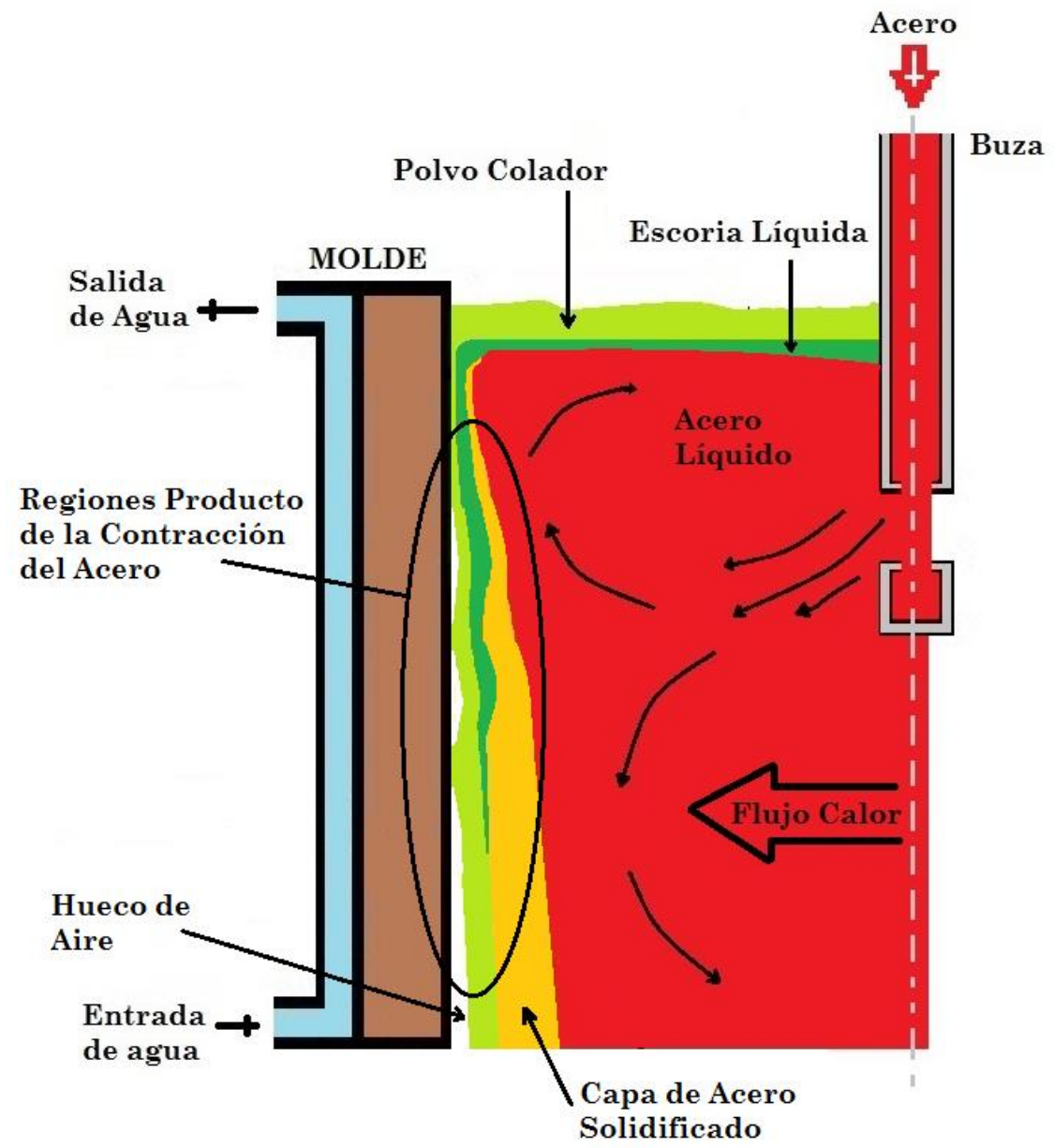

Figura 5. Formación de depresiones y de huecos de aire durante la solidificación de la costra Fuente: elaboración propia.

Los huecos de aire, producto de la contracción del acero, es de aproximadamente $0.2 \mathrm{~mm}$ (a una ubicación media de la longitud del molde) y se debe principalmente, además de la extracción de calor, a la presión ferrostática y las propiedades mecánicas de la costra solidificada. En la región de las aristas del molde, el espacio de aire formado es mayor porque el enfriamiento se produce a través de ambas caras. Mientras mayor es el hueco de aire, el flujo de calor disminuye y se favorece el sobrecalentamiento en la zona de la pieza, que pudiera ser deformada debido a la presión ferrostática y llevar a la rotura del producto [16]. El uso de moldes con conicidad uniforme y variables se han experimentado con la finalidad de reducir la brecha que se forma producto de la contracción. En este sentido, al disminuir el hueco de aire, aumentará el flujo de calor, se favorece el crecimiento del espesor de la costra sólida, se reduce el recalentamiento y disminuyen las posibilidades de formación de grietas [1], [67].

La rotura de la costra solidificada genera un problema grave que obstaculiza la producción de acero, y está relacionado con la formación de regiones delgadas en la costra por depresiones, errores operacionales o grietas, entre otros [28]. En [12] se hace un resumen sobre los problemas de calidad más importantes en la fundición de palanquillas, siendo una de las principales causas la interacción adversa entre el molde y la costra solidificada. Otra característica de importancia es la distorsión térmica que sufre la pared del molde, según [68] llega a ser máxima debajo del menisco donde el molde está más caliente y cambia dinámicamente durante el proceso, debido a variaciones en la temperatura del molde por 
efectos de la ebullición nucleada en la camisa de agua de enfriamiento y fluctuaciones en el nivel de metal. Este comportamiento termomecánico del molde tiene gran importancia en el proceso porque influye en la interacción molde y costra solidificada, que lleva a problemas en la calidad [12].

\subsection{Aceites y polvos coladores}

El molde de colada debe ser lubricado para evitar que se genere fricción entre la pared del molde y la costra solidificada, de lo contrario pueden crearse marcas y roturas en los productos de acero. La lubricación a base de aceites (de origen vegetal, aceite de colza, mineral y de parafina) mejora el deslizamiento de la barra en el molde y proporciona un buen estado superficial de la pieza [11], [69], pero su uso debe ser de estricta supervisión porque el empleo inadecuado puede causar determinados defectos superficiales en las barras [19]. En el colado con aceite comúnmente el vaciado es a chorro abierto, lo cual es particularmente problemático, porque se presentan fluctuaciones en el menisco, causadas por los fuertes gradientes de velocidad en esta región que interactúan sobre la película lubricante de aceite y provocan variaciones en la transferencia de calor del molde, generando efectos adversos en la calidad de la palanquilla colada [1]. Otra forma de lubricación es mediante polvos coladores o fundentes para molde de colada, que son escorias sintéticas usadas para cubrir el acero líquido en el molde. Están compuestos, básicamente, por $\mathrm{SiO}_{2}, \mathrm{CaO}$ y $\mathrm{Al}_{2} \mathrm{O}_{3}$, más el agregado de fundentes (óxidos alcalinos $\mathrm{NaO}_{2}$ y fluoruros $\mathrm{CaF}_{2}$ ) que controlan el rango de fusión y la viscosidad de la escoria formada. También se agrega carbón de diferentes orígenes y granulometría para regular la velocidad de fusión [1], [8], [16]. Los polvos coladores son más beneficiosos que el aceite, porque reducen mucho más la fricción, ofrecen funciones como las de dar aislamiento químico y térmico al acero líquido y permiten colar con bajas temperaturas de colada [1]. La forma y composición química del fundente influyen en su punto de fusión, viscosidad y tensión interfacial [26].

Actualmente el uso de polvos como lubricantes se ha convertido en una práctica prioritaria debido a su fuerte impacto en la calidad [8], [11], [26], su introducción manual en el molde genera variaciones en el espesor e inestabilidad en el menisco, pero, con el uso de métodos de alimentación automática se ha logrado mejorar estas condiciones [52]. De ocurrir fluctuación en el nivel o alta velocidad, puede producirse atrapamiento de escoria, pero una baja fluctuación no favorece la flotación de inclusiones y la transferencia de calor [70]. Al introducir los polvos en el molde, quedan nadando sobre la superficie del acero, formando las tres capas de la Figura 6: la capa superior es de polvo en su estado original, más abajo esta un polvo sinterizado y luego una de escoria líquida en contacto con el acero líquido. Las funciones principales son: evitar de la reoxidación del menisco de acero líquido, crear un aislamiento térmico, absorber las inclusiones que llegan a la superficie, mantener lubricado el contacto acero -solidificado-molde y ayudar a una extracción uniforme de calor [11], [26], [71]. Cuando la lubricación es con polvos, el vaciado del acero se realiza mediante BES, con la finalidad de prevenir que el polvo sea arrastrado al seno del metal por las corrientes ascendentes y mejorar potencialmente la estabilidad en el nivel del metal líquido, ya que disminuye en gran magnitud la turbulencia superficial [1], [4], [8], [19]. 


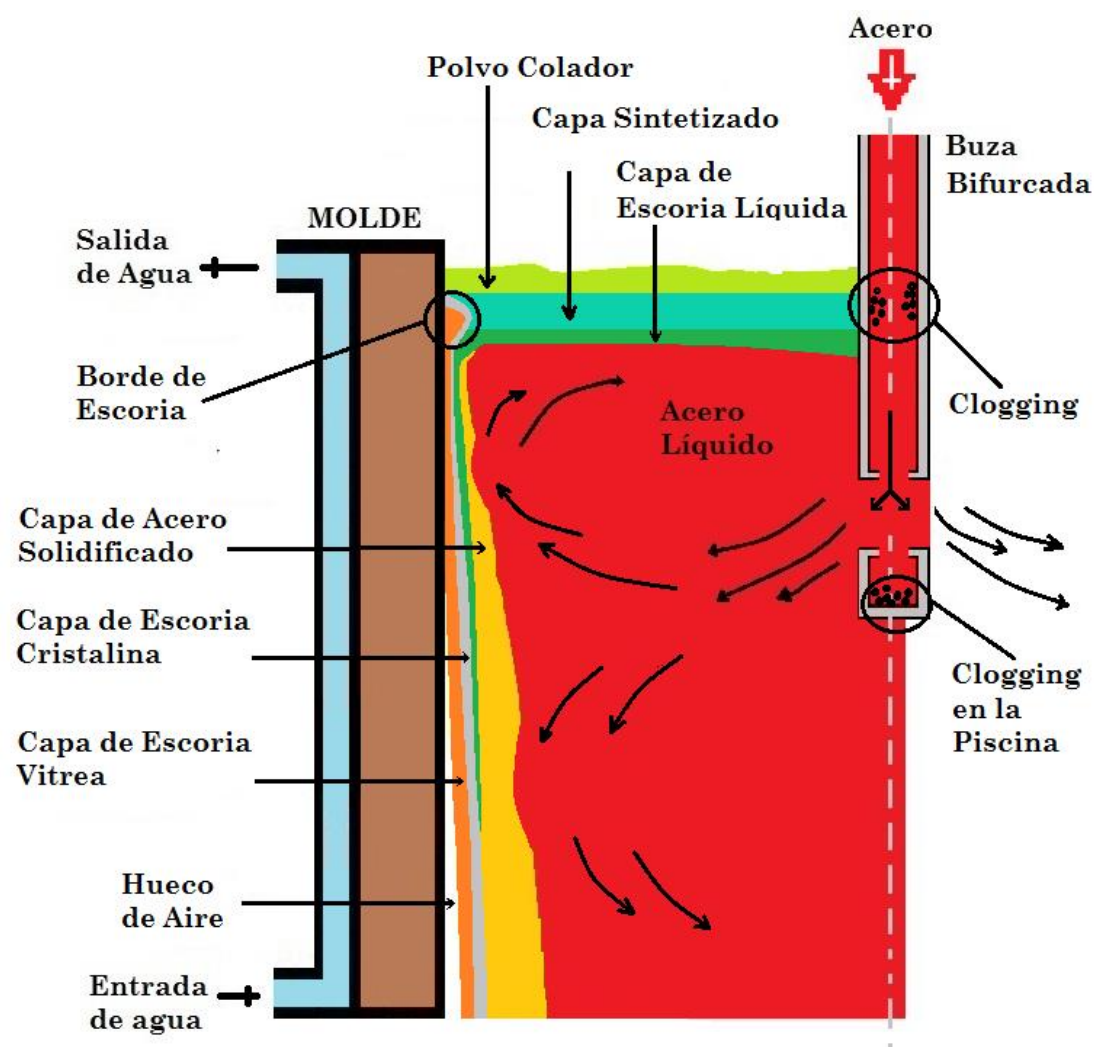

Figura 6. Comportamiento del polvo colador en el molde de colada continua. Fuente: elaboración propia.

La capa de escoria líquida, ubicada en el nivel del acero, aprovecha los movimientos de oscilación del molde para infiltrarse entre la costra de acero solidificado y la pared del molde [24], [43]. La capa de escoria próxima a la pared del molde se enfría y solidifica (como un sólido) aumentando considerablemente su viscosidad, denominada capa de escoria vítrea (de aproximadamente $2 \mathrm{~mm}$ de espesor, pero de mayor grosor encima del menisco). Mientras que, con el acero, una escoria líquida de poco espesor (1 $\mathrm{mm}$ aproximadamente) acompaña el descenso de la línea. Con el tiempo, las partes más calientes de la capa sólida y vítrea cristalizan. La escoria que se solidifica en la zona del menisco de acero da lugar a la formación de un borde de escoria, causando un abultamiento debido a la curvatura del menisco y una aglomeración debido a la variación del nivel del molde en la interfaz de escoria-sintetizado. Este borde tiene importancia en la formación de marcas de oscilación y en la transferencia de calor en el molde [1], [11], [16].

Durante la solidificación del fundente, la transferencia de calor en el molde disminuye significativamente, ya que el espesor solidificado del fundente aumenta, y la resistencia térmica crece igualmente [42]. La extracción del flujo de calor disminuye al utilizar polvos con mayor viscosidad y temperatura de fusión [16]. La viscosidad óptima de la escoria del polvo está estrechamente relacionada con el tipo de acero fundido, la velocidad de fundición y la oscilación. A menor viscosidad del polvo (aumento de su nivel de alcalinidad), mejora la lubricación y genera marcas de oscilación más pequeñas, y a mayor viscosidad, dificulta fluir entre la losa y la pared del molde. A menor grado de alcalinidad se producen marcas de oscilación más largas y profundas [72]. Es importante la optimización del polvo colador para evitar la formación de fisuras [4]. Cuando se cuelan aceros próximos al $0.1 \%$ de carbono, se requiere una elevada temperatura de cristalización en los polvos coladores, con la finalidad 
de favorecer la formación de una capa cristalina, en la que se desarrollan microgrietas que tienden a disminuir la extracción de calor en la zona del menisco [16], [19].

La composición química de los polvos es determinante en la transferencia de calor por radiación y conducción en el molde de colada [71]. De acuerdo con la teoría de la estructura iónica de las escorias líquidas metalúrgicas, el líquido iónico (fase de la formación de escoria líquida del polvo en el molde) se produce entre dos metales (la pared de cobre del molde y la superficie de acero) y crea un tipo específico de celda galvánica (en la zona cercana al menisco).

Esta celda puede provocar cambio en la composición química de la fase líquida del polvo, de manera que tiende a cambiar la condición de lubricación e influye en las marcas de oscilación [72]. Debido a la complejidad de la conductividad térmica del polvo, el tipo de fundente que debe seleccionarse en relación con el tipo de acero a colar y las condiciones de colada, es muy común que en las plantas de colada se deje a criterio del proveedor cuál debería ser el polvo adecuado; pero en muchos otros casos se aplica el método de prueba y error [8].

\subsection{Buza de entrada sumergida}

La buza sumergida (BES) es un conducto (hecho de refractario [13]) que proviene de la artesa y se prolonga en el molde para introducir el acero en fase líquida, mostrada en la Figura 1. La BES crea comúnmente una hidrodinámica en el interior del molde. En el caso de la colada de planchones se forman dos vórtices de gran tamaño en la parte inferior del molde y dos pequeños en la parte superior [20]. Dependiendo de la velocidad que trae el fluido, a la salida de la BES se forma un patrón de flujo característico que además de influir en la dinámica del molde [13], [57] afecta la formación de la costra solidificada [30]. En el flujo que sale de la BES es importante conseguir simetría, porque puede dañar la calidad del producto final y causar atrapamiento de inclusiones, turbulencia superficial anormal, atrapamiento de escoria, transporte de sobrecalentamiento insuficiente al menisco y otros problemas [73].

Entre las funciones de la BES, está la de mantener constante el nivel de acero en el molde, proteger el chorro de acero, prevenir reoxidación y evitar el atrapamiento de polvos de colada o impurezas que afectan la calidad del producto [19], [13], [74]. En los últimos años se han analizado las diferencias metalúrgicas en las piezas colada y la dinámica del fluido en el molde bajo distintos tipos de buzas. Los resultados arrojan que las BES con un agujero de salida recto (tipo tubería) son desfavorable para la eliminación de inclusiones no metálicas mediante la flotación y tiende a conducir a un menisco inactivo [75]. Mientras que, con la BES de dos agujeros bifurcada, como la utilizada en la Figura 6, trae el beneficio de obtener una alta tasa de eliminación de inclusiones, pero tienen el inconveniente de afectar la costra solidificada, haciendo que se adelgace donde impacta el chorro de acero líquido con la cara del molde [75], [76]. Con una BES que contenga cuatro agujeros con salidas en direcciones tangenciales, mejora el patrón de flujo en el molde, formándose remolinos que mantienen un crecimiento uniforme de la costra solidificada [77], [78]. Cuando la disposición de los cuatros agujeros se colocan en el molde de manera diagonal, disminuye el efecto de impacto del flujo de acero en las caras del molde, lo que es beneficioso para el crecimiento uniforme de la costra que se va formando, y esto se debe a la baja velocidad de inyección y la larga distancia de impacto a la pared del molde. La BES de cuatro puertos en comparación con la BES de dos puertos, reduce la fluctuación en el nivel del molde, mejora la flotación de inclusiones y favorece la solidificación inicial [75]. Con la BES de cinco orificios, [79] determinó que la velocidad del flujo de acero fundido en el frente de la interfaz de solidificación es mayor que para una BES de un solo orificio, independientemente de la potencia en la aplicación de fuerzas electromagnéticas. 
Las características geométricas internas de una BES modifican la fluidodinámica y el patrón de flujo en el molde [1], [30], [57], así como la distribución de velocidad en el menisco [1], [13], [57]. Sin embargo, algunas características pueden cambiar de manera natural debido a las condiciones de operación de la colada continua. Un ejemplo de esto es la modificación de la geometría de los puertos de salidas debido al desgaste de la BES ocasionada por erosión [80]. Algunos tipos de BES tienen una estructura compleja y tienden a provocar fácilmente el fenómeno clogging mostrado en la Figura 6, que se refiere a la obstrucción de las boquillas de la buza sumergida por la acumulación de material en el paso del acero entre la artesa y el molde [74], [81]. También la recirculación del flujo y la deposición de inclusiones en la piscina modifican la geometría y afectan el patrón de flujo con direcciones al molde [20], [57], [74] que influye en el adelgazamiento de la costra sólida [20], [81]. Una buena alternativa para reducir el clogging es disminuyendo la concentración de productos de desoxidación y la formación de productos de reoxidación, es decir, mejorando la limpieza del acero [74].

En [30] variaron la longitud de inmersión de una BES en un molde, así como el diseño interno (ver la Figura 7) para estudiar las fluctuaciones de presión y velocidad. Encontraron que con una menor altura del divisor (Figura 7a), se forman vórtices en la BES por la forma de separación de la capa limite en el divisor de los puertos, fenómeno que aunado a la recirculación del acero proveniente del fondo del molde provoca la falta de simetría en los chorros y oscilaciones a altas velocidades de colada, mientras que al aumentar la altura del divisor (Figura 7b), se tiende a eliminar la formación de vórtices.

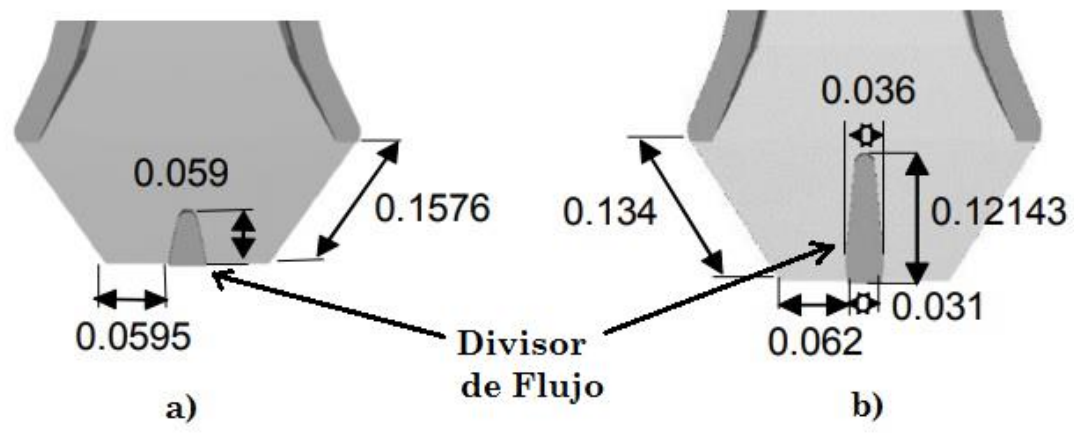

Figura 7. a) BES-original, b) BES-modificada. Fuente: [30].

También observaron que, a distinta longitud de inmersión de la BES en el molde, los chorros experimentan una variación en su patrón de flujo a medida que aumenta la velocidad de colada. En [10] concluyeron que, a medida que aumenta la profundidad de inmersión de la BES, hay una disminución en la intensidad y en la velocidad del flujo dirigido hacia la superficie libre, y que es posible recomendar un intervalo de funcionamiento entre $150 \mathrm{~mm}$ y $200 \mathrm{~mm}$ para la profundidad de inmersión de la BES. Los resultados de [82] indican que, un aumento en la velocidad de colada y una reducción en la profundidad de inmersión de la BES, conducen a la reducción del espesor de la costra solidificada. En [57] observaron en BES con dos agujeros la formación de dos recirculaciones superiores y dos inferiores, y que los chorros de acero que primero inciden en las caras estrechas del molde son los que giran hacia arriba, hacia la superficie superior, y que al cambiar las condiciones de fundición (molde más ancho, menor inmersión de la BES) se puede revertir el patrón de flujo a solo dos recirculaciones. En [20] se describió que la profundidad de la piscina es un parámetro muy importante en el diseño de la BES, debido a que influye en el tipo de patrón de flujo dentro del molde, y en la forma de que el flujo de acero sale de la BES y disipa su energía cinética. Cuando el chorro golpea directamente a las paredes angostas del molde, el espesor de la costra solidificada puede 
disminuir y afectar considerablemente la transferencia de calor por conducción. Por ello es importante controlar que el chorro disipe su energía cinética hacia las paredes más anchas.

Cuando el sobrecalentamiento es bajo y la transferencia de calor es alta, el acero puede congelarse dentro de la BES [81]. Pero, una técnica bastante importante es la generación de un flujo giratorio mediante la BES, porque tiende a producir un componente de flujo rotacional que mejora la transferencia de calor cerca del menisco y disipa el sobrecalentamiento del acero [79], así como también reduce el problema de obstrucción de los puertos laterales de la BES [55]. En [77] generaron un flujo de remolino en la dirección antihoraria en la zona superior del molde debido a la característica geométrica tangencial a las salidas de la BES, y simultáneamente, crearon un flujo de remolino por agitación magnética en el sentido de las agujas del reloj en la parte inferior del molde, logrando reducir la segregación de carbono debido al mejor efecto de disipación de sobrecalentamiento. También es de importancia, la intensa erosión de la pared exterior de la BES que entra en contacto con la escoria en el molde, a pesar que para esto se ha adaptado la composición química de la escoria de acuerdo con el tipo de acero y se ha hecho uso de recubrimientos antioxidantes en el área de contacto; sin embargo, en la industria la profundidad de inmersión de la BES es comúnmente variada para reducir el desgaste localizado y aumentar la vida útil de la BES; en otras palabras, la productividad de la planta y la calidad de la superficie de la losa de acero puede verse comprometida si no hay control de la inmersión de la BES [10].

\subsection{Segregación}

En general, la segregación se presenta porque la difusión no es capaz (durante el tiempo que dura la solidificación) de homogeneizar el sólido que se va formando en el proceso de colada, creando una heterogeneidad de tipo químico, donde resultan algunas zonas más ricas en soluto que otras [9], [40]. Durante el cambio de fase, el primer metal que tiende a solidificar tendrá el contenido más bajo de soluto, y la concentración de soluto aumentará en la fase sólida con el progreso de la solidificación [40], [63], [83], produciéndose un material con propiedades físicas y mecánicas no uniformes. El control de la segregación tiene que ver con las altas velocidades de solidificación y la evolución de la microestructura [4]. De las muchas causas de segregación en la colada continua, se mencionan la alimentación de la contracción por solidificación, los gradientes térmicos y solubles en el líquido, la fuerza impulsada por la flotabilidad, la velocidad de colada y el movimiento de granos equiaxiales debido a la nucleación heterogénea, entre otras. Se conocen dos tipos de segregación encontradas en las estructuras: la microsegregación y la macrosegregación [23], [84]. Se habla de macrosegregación cuando en el proceso de solidificación del acero, el elemento soluto se rechaza de la dendrita sólida y se enriquece en la fase líquida (siendo el soluto el menos soluble en la fase solida [85]), y con el efecto del flujo de fluidos, el soluto rechazado es arrastrado y transportado a larga distancia [23], [83]. Este tipo de segregación constituye serios problemas debido a la gran longitud de difusión requerida [23] y se presenta en forma longitudinal en la losa [43]. La mayor parte de la macrosegregación es causada por el mecanismo del flujo de líquido a través de los espacios interdendríticos en la zona líquido-sólida, y las causas de este flujo incluyen la contracción por solidificación, la convección inducida por la gravedad y el movimiento de sólidos (por ejemplo, "abultamiento") [86]. Por su parte, la microsegregación está íntimamente relacionada con la forma y crecimiento de las dendritas, además de la naturaleza de las fases de solidificación y las reacciones de precipitación, las temperaturas y velocidades de enfriamiento [43], [85]. Este tipo de segregación no constituye mayores problemas para la calidad cuando los efectos pueden ser removidos en posteriores trabajos como la homogenización [23], y se presenta de tipo lateral [43]; sin embargo, en la colada 
continua no hay tiempo para esos procesos. Para mejorar la segregación, es necesario controlar la temperatura de colada en la cuchara/artesa, la velocidad de colada y el flujo de calor en el contorno de la losa. Cuando la velocidad de enfriamiento es elevada el rechazo de soluto se incrementa de la fase sólida hacia la fase líquida [43]. Si los aceros son altamente aleados, experimentan mayor segregación [57]. En aceros con alto contenido de carbono, la segregación se crea en la línea central de palanquilla, influenciado por el sobrecalentamiento y la velocidad de colada, teniendo su origen en la zona del molde por esfuerzos térmicos, porque la pieza experimenta diferentes velocidades de enfriamiento entre su interior y el exterior [81]. En [17] se hace una descripción de los diferentes tipos de zonas de segregación que pueden presentarse en una losa de acero.

A medida que el metal se solidifica, a nivel micro, los elementos de la aleación en la zona pastosa se rechazan desde las dendritas sólidas en crecimiento hacia el líquido interdendrítico vecino [2], [85]. Este líquido se enriquece cada vez más con elementos de aleación a medida que avanza la solidificación, de manera que la segregación da como resultado una distribución de soluto no uniforme entre los brazos de dendrita [9], [43]. Al reducir el tamaño de la sección de la losa, más fina será la escala de la estructura dendrítica y la segregación asociada; por lo tanto, en este sentido, la colada continua de secciones más pequeñas es beneficiosa [63]. Una forma de mejorar la segregación es aplicando agitación magnética en forma estratégica en el molde y en el enfriamiento secundario [57]; no obstante, recientemente se ha puesto en marcha un método innovador, que consiste en la alimentación de barras consumibles de acero en el molde, para aliviar la segregación y la porosidad central de la placa. A través del cambio de fase de la barra, el sobrecalentamiento en la zona central disminuye, promoviendo la formación de granos equiaxiados que dificultan el crecimiento de la estructura dendrítica columnar [44], [87].

\subsection{Menisco y oscilación del molde}

En la Figura 8 se muestra cómo el acero líquido forma un menisco en la parte superior del molde. Los parámetros geométricos del menisco como el radio $\left(R_{m}\right)$ y altura $(h)$ afectan la formación de la costra de acero solidificada, y al mismo tiempo, la calidad de la superficie de los productos de colada [72]. La costra comienza a formarse en la superficie del menisco debido al efecto de enfriamiento del molde. Luego la costra se va enderezando a lo largo del molde, bajo la influencia de la presión ferrostática que ejerce el acero líquido [72]. En [88] se explica que, al inicio de la solidificación, la costra debe ser lo suficientemente gruesa, de lo contrario, si la costra es demasiado delgada, la punta se doblará y deformará. Si durante este proceso ocurren fluctuaciones en el nivel del metal líquido, esto afectara a la solidificación inicial debido a la dinámica del molde y la interacción con el polvo lubricante [89]. En [90] se reportó que manteniendo una variación de nivel de acero en el molde en un rango de $+/-2 \mathrm{~mm}$ (3 mm según [10]) y con una velocidad de cambio inferior a $1.5 \mathrm{~m} / \mathrm{segundos}$, mejora los aspectos de calidad y continuidad operativa. En plantas usan una regla empírica donde las variaciones de velocidad en la superficie no deben exceder del $20 \%$ [80]. Ahora bien, es conveniente el uso de un adecuado sistema de control del nivel en el molde porque con el agregado de polvos coladores tiende a ser afectado. Por ejemplo, cuando se utilizan sistemas de control con sensor radioactivo, el sensor percibe un aumento en el nivel de acero al adicionar el polvo colador, y en realidad el nivel del menisco de acero no ha cambiado [52], [90]. 


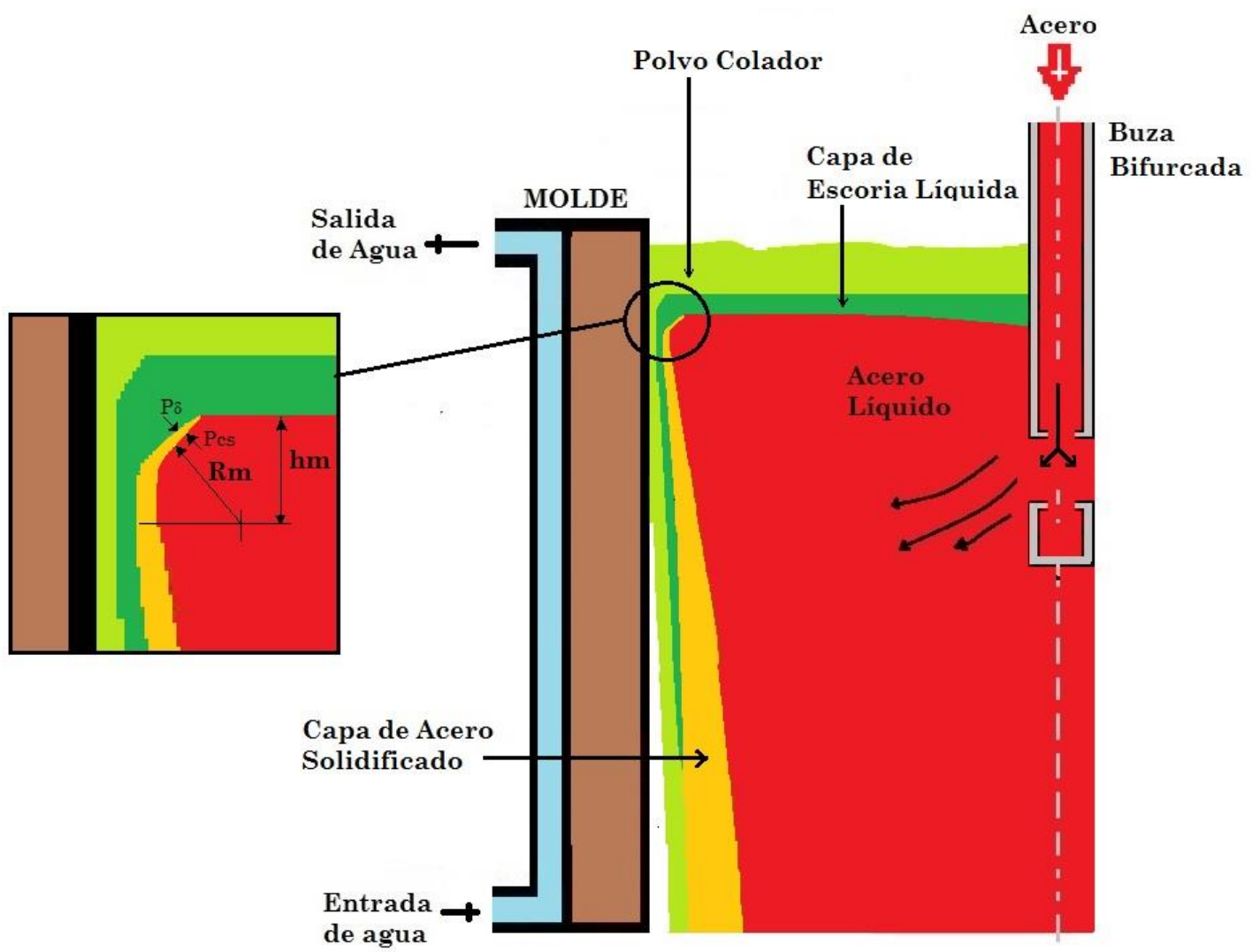

Figura 8. Menisco convexo de acero líquido cerca de la pared del molde. Fuente: elaboración propia.

Respecto al radio interno de la esquina del molde, éste posee gran influencia en el origen de a) grietas externas ubicadas a lo largo de las aristas y b) grietas internas en las secciones transversales próximas a las esquinas. Cuando los radios son menores a $6 \mathrm{~mm}$, disminuyen la ocurrencia de las grietas en las aristas, pero que a su vez fomentan las grietas internas; en forma inversa, a medida que aumenta el radio interno de la esquina del molde, las grietas internas disminuyen, pero a su vez se incrementan las grietas externas ubicadas en las aristas [67]. En [42] determinaron que en la esquina del molde el espesor de la capa solidificada es mayor que en el centro de las caras, y que el fundente solidifica primero en el vértice que en el centro de las caras. Y en las esquinas y en la parte media de la cara en las palanquillas cerca del menisco se originan grietas superficiales, tanto transversales como longitudinales, y esto se ha relacionado a las marcas de oscilación [12], [52], [71].

Debido a la tensión superficial $\left(P_{\delta}\right)$, la presión ferrostática $\left(P_{c S}\right)$ y la escoria fundida, el radio del menisco $\left(R_{m}^{Z}\right)$ se determina por [72] en (8):

$$
R_{m}^{Z} \cong 1,699 \sqrt{\frac{\sigma_{c s-z}}{g \cdot\left(\rho_{c s}-\rho_{z}\right)}}
$$

Dónde $\sigma_{c s-z}$ es la tensión de interfase en el límite entre el acero y la fase líquidos del polvo de fundición, $\rho_{z}$ es la densidad de la fase líquida del polvo de fundición, $\rho_{c s}$ es la densidad del acero líquido y $g$ es la aceleración de gravedad. En (8) se demuestra que existe la posibilidad de afectar los parámetros del menisco por medio de la fase líquida del polvo de fundición. 
Un radio menor del menisco significa una altura más baja y una menor probabilidad de formar defectos superficiales como pliegues, rizados y fracturas [72].

Durante la colada es importante que el patrón de flujo del molde entregue al acero fundido, en la región del menisco, suficiente sobrecalentamiento durante las primeras etapas críticas de solidificación [21], [57]. Y, para mejorar la transferencia de calor en el menisco, es posible usar una conicidad en el molde poco profunda para mantener a la costra solida pegada al molde. Pero si las condiciones de lubricación no son las adecuadas, y con la presencia de una costra sólida delgada, puede provocarse el deterioro de la costra (desgarro y filtrado) en aceros con alto contenidos de carbono, y esto se ha relacionado a las fluctuaciones del nivel de acero durante el proceso de fundición [12]. Otro efecto que se le suma al menisco y la formación de la costra es la oscilación del molde, la cual ayuda a que la capa de acero cure las fisuras superficiales y las porosidades, así como también contribuye en la distribución de temperatura dentro del molde [25]. El movimiento oscilatorio también reduce la fricción entre la pared del molde y la costra solidificada de acero, facilitando el despegue entre ellos, y a esto lo ayuda la introducción de polvos coladores [4], [14], [91]. Pero la fuerza de fricción entre la pared del molde y el acero fundido es afectada por el efecto de la viscosidad del fluido [92].

Producto del movimiento del molde, se crean marcas de oscilación en los productos de colada [89], que son ondulaciones formadas en la superficie del material paralelas entre sí y perpendiculares a la dirección de colada [69], [93]. La profundidad de la marca de oscilación depende de los polvos colador, la carrera del molde, la frecuencia de oscilación y la velocidad de colada [4], [93], [94]. En [92] observaron que, a mayor frecuencia de oscilación, mayor es el impacto en las fluctuaciones, aumentando la altura de las olas y la velocidad en la superficie. En [69] determinaron que, cuanto mayor sea la tensión interfacial, más profundas y anchas serán las marcas. En (9) y (10) se definen la distancia entre las marcas de oscilación, $l$, y la profundidad, $d$, en las superficies de la losa [69], [72]:

$$
\begin{gathered}
l=\frac{V_{c}}{f} \\
d=0,065\left(1,145^{S}\right)\left[200\left(0,9^{S}\right)\right]^{t_{N}}
\end{gathered}
$$

Donde $V_{c}$ es la velocidad de colada, $f$ la frecuencia de oscilación, $t_{N}$ es el tiempo de desmolde negativo y $S$ la carrera del molde. Es de notar que la frecuencia de oscilación varía en función de los cambios de la velocidad de colada [14]. En la Figura 9 se muestran los movimientos hacia arriba (desmolde positivo, $\mathrm{t}_{\mathrm{P}}$ ) y hacia abajo (desmolde negativo, $\mathrm{t}_{\mathrm{N}}$ ) que realiza el molde según la curva roja $\left(\mathrm{m}_{\text {disp }}\right)$. Durante $\mathrm{t}_{\mathrm{N}}$ el molde desciende más rápido $\left(\mathrm{V}_{\mathrm{m}}\right)$ que la velocidad de colada $\left(\mathrm{V}_{\mathrm{C}}\right)$ presentado por un cuadro gris, mientras que tp se refiere al resto del ciclo. En el periodo de tp se efectúa el despegue de la costra de acero de la pared del molde [15], [24], [94], y en el periodo de $t_{N}$, el polvo de colada se infiltra entre la costra y el molde [93], [94]. La diferencia de velocidad entre el molde y la losa $\left(\mathrm{V}_{\mathrm{m}}>\mathrm{V}_{\mathrm{C}}\right)$ trae como resultado mejoras en la calidad de la superficie de las piezas de fundición, debido a que la costra se desprende de las paredes del molde bajo la influencia de pequeño esfuerzo de compresión y no se somete a esfuerzos de tracción [72]. 


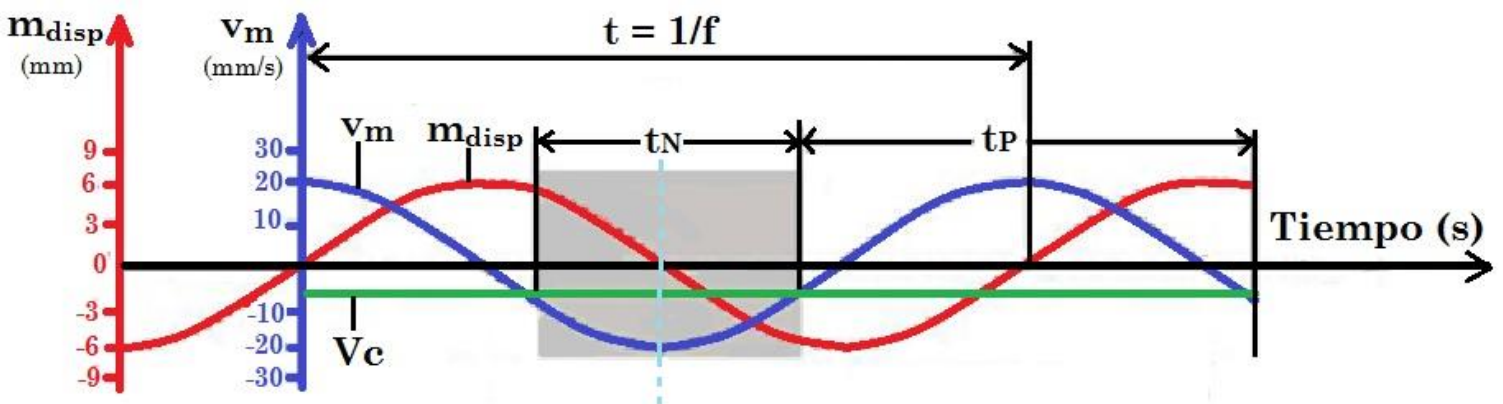

Figura 9. Vibraciones en el molde de colada continua. Fuente: elaboración propia.

Si bien la oscilación del molde es una necesidad para la colada continua, también es un problema debido a su influencia en los defectos superficiales, en el cambio en la distribución de temperatura dentro del molde y en el origen de fisuras transversales [22]. A la oscilación también se le atribuye la superposición de muchos fenómenos físicos variantes en el tiempo en la zona del menisco [6], tales como fluctuaciones en la presión del acero líquido, fusión del polvo de fundición, variaciones en la tensión interfásica escoria-metal, disipación de calor y deformaciones en la costra solidificada durante las oscilaciones [95]. Otras características de importancia son los ganchos y marcas de oscilación que se forman debido a los muchos fenómenos físicos interactivos, descritos anteriormente, que varían con el tiempo cerca del menisco donde la superficie del acero fundido se encuentra con la pared del molde. Estos eventos juntos determinan la forma y el tamaño de los ganchos debajo de la superficie y sus correspondientes marcas de oscilación [93]. También se ha explicado [69], [93], [94] que los parámetros de mayor influencia en la formación de marcas de oscilación es el tiempo de desmolde negativo y positivo. El incremento de $t_{N}$ está acompañado por una mayor profundidad en las marcas de oscilación y un menor espesor de la capa de escoria entre la losa de acero y el molde [60], [72], y la disminución de tp se asocia a un menor consumo de polvo de fundición y menor profundidad de las marcas de oscilación [72]. Las marcas de oscilación son consideradas defectos porque la segregación y el agrietamiento tienden a ocurrir en la base de la marca, y la gravedad del problema aumenta al aumentar la profundidad de las marcas [15]. Por esto es importante conseguir marcas de oscilación poco profundas, debido a que allí se pueden encontrar cristales columnares, los cuales se consideran indeseables en las capas subterráneas por su responsabilidad en la fisuración del acero durante el proceso de laminación [94], además que un aumento en la profundidad de las marcas de oscilación reduce la transferencia de calor en el molde [6]. Para minimizar las marcas de oscilación, se recomienda que el tiempo de desmolde negativo debería estar entre 0.08 s y $0.15 \mathrm{~s}$ [4], [72] y, según [69] el tiempo de desmolde negativo, debe tener valores típicos de $0.2 \mathrm{~s}-0.3 \mathrm{~s}$ para evitar que la costra de acero se pegue del molde. En [93] se muestran detalles de los mecanismos para la formación de marcas de oscilación, así como otra característica importante asociada a la oscilación, conocida como "gancho", que es una característica en la microestructura sub-superficial que a menudo acompaña a una marca de oscilación, donde su severidad aumenta con la disminución del contenido de carbono, lenta oscilación y menor velocidad de colada.

Aunque la oscilación del molde es normalmente periódica en el tiempo, esto no significa en general que las marcas que se forman sean idénticas y estén espaciadas periódicamente [51]; en la práctica se ha observado que no son idénticas [89]. En [91] se ha experimentado el modo de oscilación no sinusoidal del molde para determinar su efecto sobre la infiltración de escoria de lubricación y el crecimiento inicial de la costra solidificada de acero cerca del 
menisco. La investigación [91] consideró un nuevo parámetro de oscilación llamado relación de modificación (a), que se refiere al grado de asimetría de la oscilación no senoidal.

Los resultados se muestran en la Figura 10, y en ella se observa que los vectores de la escoria líquida se infiltran entre el molde y la costra de acero solidificada formando un canal.

Durante el descenso del molde, el flujo de escoria entra fuertemente al canal con una distribución de velocidad uniforme (casos a, b, c). Cuando el molde está a la mitad de la carrera (en el centro del cuadro gris $\left(t_{N}\right)$ de la curva roja mostrada en la Figura 9), un gran volumen de escoria líquida se expulsa del canal para regresar a la superficie (casos d, e, f), haciéndose más fuerte a elevados valores de a. Este fenómeno es causado por el borde de la costra de acero sólida que desciende juntamente con el molde de cobre, imponiéndose una fuerte presión sobre la escoria líquida. Sobre la forma de la punta de la costra solidificada, en [69] se explicó que se puede conducir calor en la dirección $\mathrm{Z}$, haciendo que crezca hacia arriba de forma parabólica, semicirculares o más planas, y que, para hacer un análisis preciso sobre este mecanismo, debería determinarse el perfil de temperatura en la superficie y la variación del grosor de la costra.
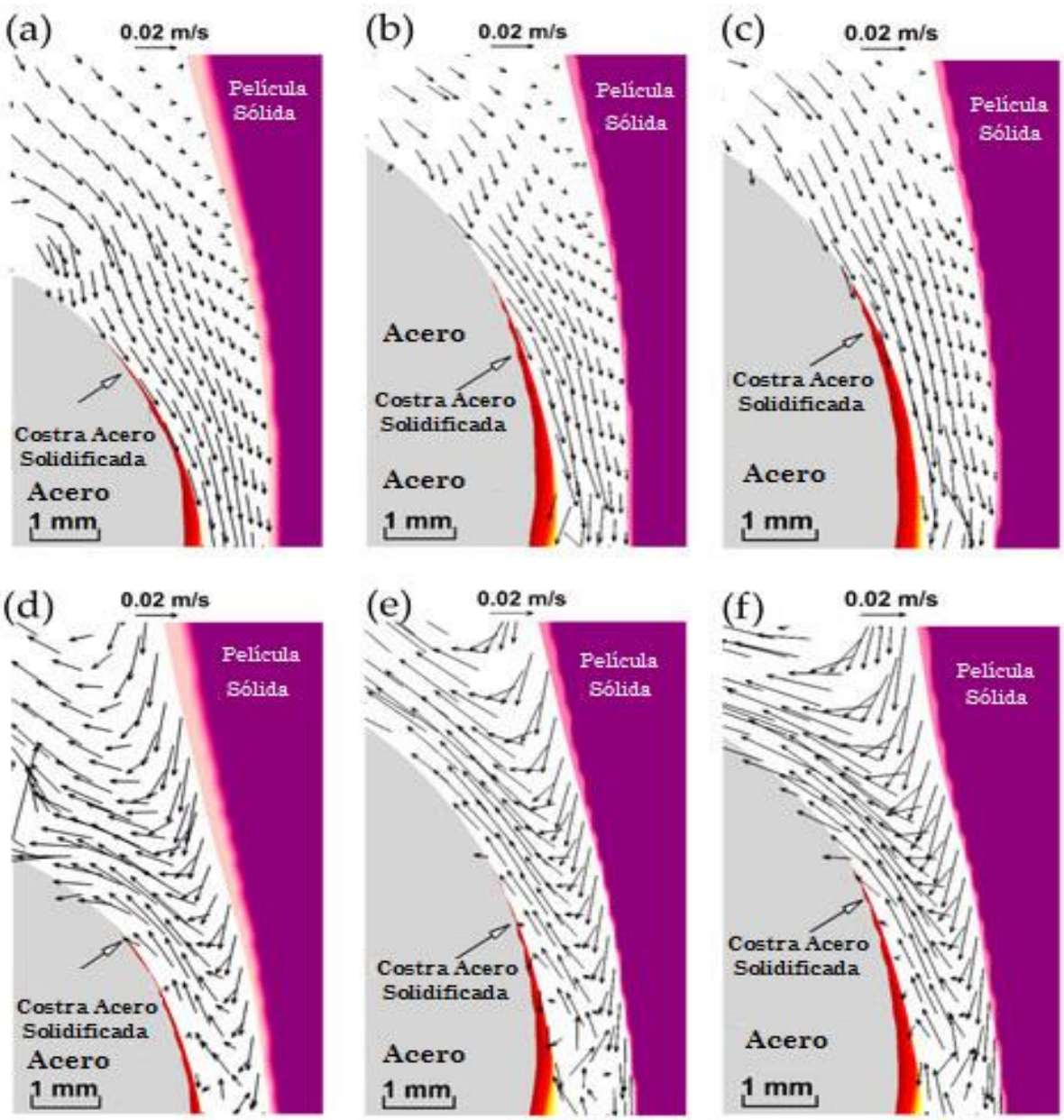

Figura 10. Infiltración de escoria en el menisco en diferentes momentos para una oscilación no senoidal: (a) $t_{1}, \alpha=0.2$, (b) $t_{1}$, $\alpha=0.5$, (c) $\mathrm{t}_{1}, \alpha=0.8$, (d) $\mathrm{t}_{2}, \alpha=0.2$, (e) $\mathrm{t}_{2}, \alpha=0.5$, y (f) $\mathrm{t}_{2}, \alpha=0.8$. Fuente: [91]. 


\subsection{Agitación electromagnética}

Los campos electromagnéticos en la colada continua se utilizan para frenar y para agitar el metal líquido en el molde y en la zona de enfriamiento secundario [70]. Con el frenado se logra reducir la variación del flujo, disminuir la velocidad, aumentar la temperatura cerca del menisco, disminuir la diferencia de temperatura en el molde y reducir el impacto del flujo cerca de la pared estrecha del molde, mientras que con el uso de la agitación se contribuye a una gran tasa de inclusión flotante, una distribución uniforme de la temperatura, una distribución homogénea de solutos, una costra solidificada uniforme y una alta calidad del producto colado de acero [55],[70]. Los agitadores magnéticos en el molde producen movimientos de giro en la fracción del metal líquido, generando desplazamientos desde el centro de la pieza hacia la intercara de solidificación. Este efecto aumenta la transferencia de calor y tiende a eliminar el sobrecalentamiento [43], [89], [96]. También ayuda a homogeneizar la composición química del líquido [57], impulsando al metal a ocupar espacios interdendríticos, minimizando los problemas derivados del microrrechupe y la macrosegregación [16], [43]. Además, favorece el crecimiento uniformemente la costra solidificada, y contribuye en reducir la segregación de la línea central y la porosidad [79], [83], [96], [97]. Aunado a esto, provoca el afino de grano mediante la destrucción de la estructura dendrítica en la fase inicial [7], [96], aumenta la proporción de granos equiaxiales en la zona central del producto colado [39], [79], homogeniza la temperatura del menisco, estabiliza la solidificación inicial y limpia la capa superficial, produciendo grandes beneficios en la calidad externa e interna del acero colado bajo cualquier condición operacional [57], [96].

En [98] observaron que, sin aplicar agitación electromagnética en el molde, el acero fundido presenta velocidad radial en la sección transversal horizontal, desde el centro hacia el exterior, y con la aplicación de agitadores electromagnética, el fundido fluye en remolino en la transversal horizontal. Encontraron que el acero líquido se expande cuando aumenta la intensidad de corriente, debido a que aumenta la fuerza electromagnética tangencial y radial sobre el acero. En [98] también determinaron que al aumentar la intensidad de la corriente de 0 A hasta $300 \mathrm{~A}$, la relación central de cristal equiaxial del lingote aumento un $10 \%$, este efecto es bueno para el refinamiento de la estructura solidificada y para la segregación central. En [85] desarrollaron un modelo matemático multifísico en 3D con agitación magnética: los resultados mostraron que con la aplicación de $200 \mathrm{~A}$ se forman dos remolinos horizontales, que hacen que la zona de recirculación superior se alargue en la dirección de colada y se promueva una segunda zona de recirculación a la salida del molde, mostrado en la Figura 11a. En Figura 11b observaron que a la intensidad de corriente de 200 A la penetración del flujo en el molde se hace menor tomando como referencia la salida de la BES, que la velocidad del fundido en la posición de instalación del sistema electromagnético se hizo mayor por el efecto magnético y la velocidad del flujo en el frente de solidificación aumento, lo que significa que los efectos de lavado del acero fundido en la capa solidificada se vuelven más significativos. En la Figura 11c, mostraron cómo la zona de alta temperatura del acero se desplaza hacia la parte superior del molde debido al efecto de agitación electromagnética que inhibe el impacto de la corriente de inyección con la alta temperatura de la BES. Hubo un aumento en el gradiente de temperatura en el frente de solidificación, lo cual es beneficioso para la extracción de calor a través del molde refrigerado. Y en la zona alta del molde, al igual que a la salida, la temperatura tuvo una disminución, por lo que se presume que el molde refrigerado extrajo más calor del fundido. 


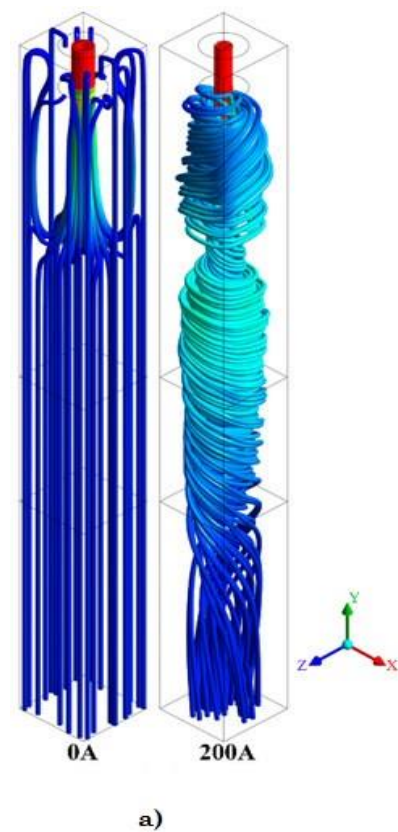

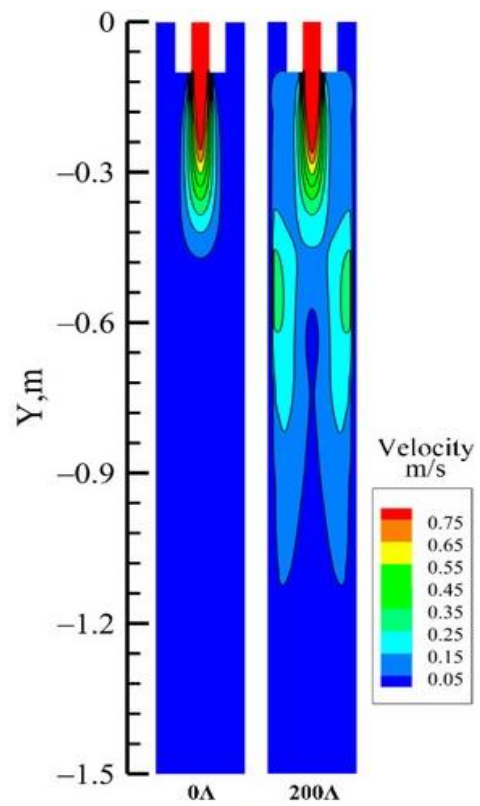

b)

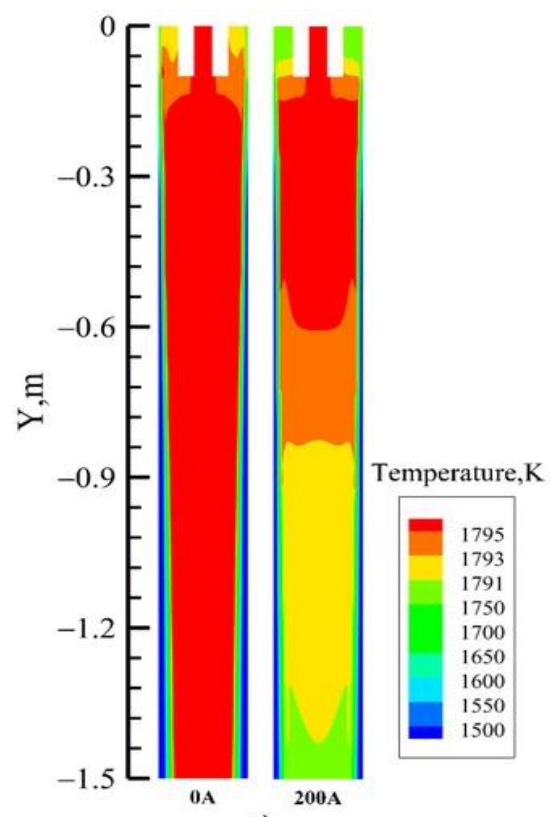

c)

Figura 11. Efecto de las fuerzas electromagnéticas en a) líneas de flujo de fluido, b) contornos de velocidad, y c) perfil de temperaturas. Fuente: [85].

A pesar de que la agitación magnética mejora la calidad del producto colado, esto generan altos costos en equipos y en electricidad, y, por consiguiente, incrementa el valor del producto de acero [55]. En las últimas décadas el uso de agitación y frenado de forma combinada es tendencia en el desarrollo de tecnología de control del flujo de acero en el molde de colada continua [57], [70].

\subsection{Perspectivas y recomendaciones}

La colada continua representa hoy el proceso más importante para la producción de acero y, desde un punto óptico, los autores ven la evolución del proceso en buen sentido, escalando en el rendimiento de las instalaciones, en mejoras de la calidad de los productos y mayor productividad. Sin embargo, en el proceso hay complicaciones debido a la concurrencia de los fenómenos que tienen lugar en el molde y a ciertas condiciones de funcionamiento. Debido a esto, hay muchos desafíos por vencer, y seguramente con el correr del tiempo, aparecerán otros nuevos, sobre todo en la búsqueda de estrategias para minimizar la aparición de defectos en el acero que tienen origen en el elemento más crítico de la colada (el molde). Se recomienda no bajar la intensidad en la búsqueda de conocimientos y soluciones, más bien unir esfuerzos para seguir contribuyendo en las mejoras del acero, debido al rol que desempeña este material en las distintas áreas de aplicación. Los trabajos futuros deben hacer un mayor uso del modelado donde se involucren múltiples modelos físicos, aprovechando el crecimiento exponencial de los recursos informáticos y el enriquecimiento del conocimiento sobre el proceso y los problemas que se presentan en él. Hay que desarrollar nuevos modelos en los cuales se desprecien cada vez menos la física, abordando una amplia gama de fenómenos ajustados aún más a la realidad y con gran precisión, sin olvidar que los resultados de los modelos deben llevarse a la planta real, donde se verifique si se logran las mejoras, por ejemplo, la reducción de los defectos en el acero que impliquen una mayor calidad. 


\section{CONCLUSIONES}

Se ha realizado una revisión amplia de lo que ocurre en el molde de la colada continua de acero para brindarle a la comunidad académica una visión sobre el comportamiento termofluidodinámico del metal. Se destacan los siguientes aspectos:

El molde es una zona de alta complejidad por la concurrencia de los fenómenos de transferencia de calor, flujo de fluidos y cambio de fase. Estos fenómenos influyen en la calidad de los productos colados, induciendo la aparición de defectos como grietas, marcas, segregación e inclusiones. Reducir los defectos no ha sido tarea fácil para los investigadores, a pesar de que se han obtenidos algunos éxitos con ciertas estrategias que han consistido en: obtener las mejores condiciones de flujo de fluidos y transferencia de calor en el molde, aplicar de manera correcta métodos de agitación electromagnética, conseguir una adecuada oscilación del molde, obtener el mejor diseño de la BES y seleccionar correctamente los parámetros del lubricante. Sin embargo, aún falta mucho por avanzar en la búsqueda de conocimientos que permitan la comprensión de la dinámica que ocurre en el molde para disminuir la aparición de defectos.

Los defectos graves en la colada continua de acero, como las grietas y depresiones superficiales, a menudo están relacionados con el comportamiento termomecánico durante la solidificación en el molde. Cuando las grietas son longitudinales se le asocia a flujo turbulento, variación en el nivel del menisco, alta velocidad de colada y comportamiento inadecuado del polvo colador, mientras que si las grietas son transversales se le agrega la profundidad de las marcas de oscilación y la fricción de la capa solidificada con el molde. Los defectos de fisuras internas se relacionan a enfriamientos bruscos, y los defectos de inclusiones están relacionados con la metalurgia secundaria, pero en el molde son removidos aprovechando los patrones de flujo. Los defectos de macrosegregación se relacionan con la contracción en la solidificación, la convección inducida por la gravedad, el movimiento de sólidos (abultamiento), temperatura de colada, la velocidad de colada y el flujo de calor en el contorno de la pieza.

Durante la formación de la capa solidificada de acero, el máximo flujo de calor que se extrae en el molde corresponde al sitio donde inicia la solidificación, es decir, en el menisco, y la principal resistencia encontrada al flujo de calor se debe a la interfaz acero solidificadomolde por la formación de un hueco de aire que tiene su origen en la contracción del acero y la deformación del molde. En esta brecha, el polvo colador se infiltra formando diferentes capas (vítreo, cristalino o líquido), producto del contacto con el molde refrigerado y el acero líquido. Las capas juegan un papel importante en la transferencia de calor, y el aporte de cada una de ellas dependerá de su espesor y conductividad, que, a su vez, dependen del perfil de velocidad, temperatura de cristalización, viscosidad y estado. El flujo de agua que enfría al molde no es menos importante, también tiene su responsabilidad en el proceso porque si las "caras frías" se calientan demasiado, puede producirse ebullición, lo que ocasionará variabilidad en la extracción de calor y defectos acompañantes.

\section{AGRADECIMIENTOS}

Este artículo no contó con apoyo económico. Se presenta en el marco de la Tesis Doctoral en Ciencias de los Materiales denominado: "Estudio de la termofluidodinámica en un molde de colada continua de acero mediante dinámica de fluido computacional", en el Instituto de Investigación en Biomedicina y Ciencias Aplicadas de la Universidad de Oriente 
(IIBCAUDO), Venezuela. Se agradece el apoyo de los profesores del programa del doctorado del IIBCAUDO.

\section{CONFLICTOS DE INTERÉS DE LOS AUTORES}

No hay conflicto de interés que declarar por parte de los autores.

\section{CONTRIBUCIÓN DE LOS AUTORES}

Yordy González-Rondón: aportó en el diseño de ideas abstractas del tema de colada continua, determinando como zona de interés el molde. Por otra parte, en el desarrollo de la investigación contribuyó en la redacción del manuscrito, seleccionó los aspectos a tratar en el molde de colada continua y realizó las Figuras para una mejor compresión.

José Eduardo Rengel- Hernández: definió algunos aspectos en el molde de colada continua, se encargó de guiar la revisión en lo termofluidodinámico y aportó en la revisión final y redacción del texto al idioma inglés.

\section{REFERENCIAS}

[1] A. Nájera Bastidas, "Análisis del flujo de fluidos y transferencia de calor sobre la calidad de palanquillas de acero", (Tesis Doctorado), Instituto Politécnico Nacional, México, 2010. URL

[2] R. Mannheim, "Introducción general a la colada continua", Revista Remetallica, no. 5, pp. 28-38. 1983. URL

[3] J. Calvo Muñoz, "Efecto de los elementos residuales e impurezas en la ductilidad y mecanismos de fragilización en caliente de un acero de construcción 0,23C - 0,9Mn - 0,13Si”, (Tesis Doctoral), Departamento de ciencia de los materiales e ingeniería, Metalurgía, Universitat Politècnica de Catalunya, Barcelona, 2006. URL

[4] B. Flores Garza, "Descripción del proceso de colada continua mediante CFD", (Tesis de maestría), Universidad Autónoma de Nuevo León, 2010. URL

[5] J. Coley Zapata, "Fundición continua, una oportunidad para mejorar la calidad de los hierros", Revista metalactual.com. Procesos, pp. 10-17. 2010. URL

[6] R. Kumar, "Computational Fluid Dynamic (CFD) simulation for continuous casting process of steels", (Tesis de Maestría), Department of Metallurgical and Materials Engineering, National Institute of Technology Rourkela, 2015. URL

[7] M. Aballe, "Colada continua y semicontinua de productos industriales", en Conference: $2^{a}$ Jornada de Ciencia y Tecnología de Materiales. Barcelona 1992. URL

[8] A. Ramírez Cruz; O. Hernández Nava; A. Aldama Moreno; M. Ramírez Vargas, "Caracterización de fundentes para molde de colada continua de acero", Acta Universitaria, vol. 17, no. 1, pp. 52-58. 2007. URL

[9] I. Hahn; M. Schneider; J. Terhaar; J. Jarolimeck; R. Sauermann, "Quality Prediction of Cast Ingots". International Conference on Casting, Rolling and Forging ICRF, 2012. URL

[10] F. Saldaña-Salas; E. Torres-Alonso; J. A. Ramos-Banderas; G. Solorio-Díaz; C. A. Hernández-Bocanegra, "Analysis of the Depth of Immersion of the Submerged Entry Nozzle on the Oscillations of the Meniscus in a Continuous Casting Mold”, Metals, vol. 9, no. 5, pp. 596, May. 2019. https://doi.org/10.3390/met9050596

[11] J. Romo Castañeda, "Estudio de la formación de especies mineralógicas en el molde de colada continua de planchón delgado de acero", (Tesis de maestría), Instituto politécnico nacional. Ciudad de México, 2011. URL

[12] S. Kumar, "Mould thermal response and formation of defects in the continuous casting of steel billets", (Tesis Doctoral), Department of Metals and Materials Engineering, The University of British Columbia. January 1996. URL

[13] M. El-Anwar; G. Megahed; M. Bedewy; M. El-Sherbiny; N. Chazly, "Simulation of Fluid Flow in Thin Slab Casting Process", Conference: 9th International Mining, Petroleum, and Metallurgical Engineering Conference (MPM), Cairo University, 2005. URL 
[14] J. Belisario, "Evaluación de la efectividad del proceso de colada en la reducción de defectos de salpicaduras en las palanquillas producidas en la acería 150 TM de Sidor", (Tesis de grado), Universidad Nacional Experimental de Guayana, 2011. URL

[15] K. Mills; P. Ramirez; P. Lee; B. Santillana; B. Thomas; R. Morales, "Looking into continuous casting mould", Ironmaking and Steelmaking, vol. 41, no. 4, pp. 242-249, May. 2014. https://doi.org/10.1179/0301923313Z.000000000255

[16] C. Cicutti, "Transferencia de calor en la colada continua de aceros, I parte, el molde", Revista Metalurgia, Madrid, vol. 33, no, 5, pp. 333-344. 1977. https://doi.org/10.3989/revmetalm.1997.v33.i5.846

[17] J. L. Enríquez Berciano; E. Tremps Guerra; S De E. de Bengy; D. Fernández Segovia, Colada del Acero, Monografías sobre Tecnología del Acero, Parte II. Madrid. 2009. URL

[18] V. Chang; P. Bolsaitis, "Simplified model for heat transfer and solidification in continuous casting", Latin American Journal of Metallurgy and Materials, vol. 2, no. 2, pp. 130-138,1982. URL

[19] M. A. Clavijo, "Consideraciones sobre la colada continua del acero", Boletín de la Sociedad Española de Cerámica y Vidrio, vol. 13, no. 4, jul. 1974. URL

[20] C. Real; L. Hoyos; F. Cervantes; R. Miranda; M. Palomar; J. González, "Influencia de la geometría de la buza sobre la transferencia de calor en un molde de colada continua de acero", $8^{\circ}$ Congreso Iberoamericano de Ingeniería Mecánica, Cusco, 2007. URL

[21] B. G. Thomas, "Modeling of Continuous Casting". Chapter 5. The AISE Steel Foundation, Pittsburgh, PA. 2003. URL

[22] B. A. Pereira; J. A. Castro; A. J. Da Silva; J. A. Duran, "Modelado del proceso de colada continua de aceros libres de intersticios", Inf. tecnol, vol. 21, no. 6, 2010. http://dx.doi.org/10.4067/S0718-07642010000600002

[23] D. Pengfei, "Numerical modeling of porosity and macrosegregation in continuous casting of steel", (Thesis Doctor) University of Iowa, 2013. https://doi.org/10.17077/etd.h7xxkots

[24] X. B. Zhang; W. Chen; L. Zhang, "A coupled model on fluid flow, heat transfer and solidification in continuous casting mold", China Foundry. vol. 14, no. 5, pp. 416-420, Sep. 2017. https://doi.org/10.1007/s41230-017-7171-2

[25] J. A. de Castro; B. Amaral Pereira; R. Sampaio de Souza; E. Mendes de Oliveira; I. L. Ferreira, "Numerical study of turbulent flows and heat transfer in coupled industrial-scale tundish of a continuous casting material in steel production", Numerical Simulations in Engineering and Science, Chapter 16, 2018. URL

[26] A. Cwudzińsk; J. Jowsa; P. Przegrałek, "Interaction of liquid steel with mould flux in continuous casting bloom mould - numerical simulations and industrial experiences", Arch. Metall. Mater., vol. 61, no 4, pp. 2013-2020. 2016. http://doi.org/10.1515/amm-2016-0325

[27] J. K. Brimacombe; I. V. Samarasekera, "Fundamental Analysis of the Continuous Casting Process for Quality Improvements", Indo-US Workshop on Materials Processing, 179-222, 1988.

[28] S. Kumar, "An Expert System to Diagnose Quality Problems i n the Continuous Casting of Steel Billets", (Tesis Maestría), Department of Metals and Materials Engineering, The University of British Columbia, Vancouver, Canada, 1991. https://dx.doi.org/10.14288/1.0078575

[29] J. K. Brimacombe; K. Sorimachi, "Crack Formation in the Continuous Casting of Steel", Metallurgical Transactions B., Sep. 1977, pp. 489-505. https://doi.org/10.1007/BF02696937

[30] H. Arcos Gutierrez; G. Barrera Cardiel; R. Escudero García, "Simulación matemática para la optimización del patrón de flujo entregado por una buza para el molde de colada continua de planchón delgado", Revista Materia, vol. 23, no. 2, jul. 2018. http://dx.doi.org/10.1590/s1517-707620180002.0447

[31] M. Vynnycky, “Continuous Casting”. Metals, vol. 9, no. $^{2}$, $\quad$ pp. $\quad 643$, Jun. 2019. https://doi.org/10.3390/met9060643

[32] J. Guirao-Goris; A. Olmedo Salas; E. Ferrer Ferrandis, "El artículo de revisión”. Revista Iberoamericana de Enfermería Comunitaria, en prensa. 2007. URL

[33] M. Cué Brugueras, G. Díaz Alonso; A. Díaz Martínez; M. de la C Valdés Abreu, "El artículo de revisión”. Revista Cubana de Salud Pública; vol. 34, no. 4, dic. 2008. URL

[34] T. Saracevik; J. B. Wood, "Consolidation of information: a handbook on evaluation, restructuring and repackaging of scientific and technical information”. Pilot edition. PGI-81WS/ 16, 1981. URL

[35] L. Cortés-Rico; G. Piedrahita-Solórzano, "Interacciones basadas en gestos: revisión crítica”, TecnoLógicas, vol. 22, pp. 119-132, 2019. https://doi.org/10.22430/22565337.1512

[36] J. D. Ospina-Correa; J. G. Osorio-Cachaya; Á. M. Henao-Arroyave; D. A. Palacio-Acevedo; J. Giraldo-Builes, "Retos y oportunidades para la industria minera como potencial impulsor del desarrollo en Colombia", TecnoLógicas, vol. 23, no. 50, 2021. https://doi.org/10.22430/22565337.1683

[37] B. Thomas; G. Li; A. Moitra; D. Habing, "Analysis of Thermal and Mechanical Behavior of Copper Molds during Continuous Casting of Steel Slabs”, 80th Steelmaking Conference, Chicago, IL, Apr, 13-16. ISS Herty Award, 1997. URL 
[38] S. Bockus, "Regulation of heat transfer in the horizontal continuous casting moulds", Proceedings of the 4th WSEAS Int. Conf. on Heat Transfer, Thermal Engineering and Environment, Elounda, Greece, pp. 94-98, 2006. URL

[39] J. P. Pulgar Hormazábal, "Perfil de solidificación de acero bajo distintas condiciones operacionales en colada continua de palanquillas", (Tesis de grado), Universidad de Concepción, Facultad de Ingeniería, 2018. URL

[40] R. J. Chung Carrero, "Efecto del enfriamiento del líquido y la solidificación primaria en la aleación Al0,55\%Fe-0,37\% Si”, (Tesis de grado), Universidad Simón Bolívar, Sartenejas, 2004. URL

[41] M. G. Shen; Y. J. Liu; X. L. Zhu; Z. Y. Xiao; Y. C. Liu, "Study on the influence of new riser structure on the quality of steel ingot", Metalurgija, vol, 58, no. (1-2), pp. 47-50. 2019. URL

[42] J. Barco, J. Palacios; C. Ojeda; M. Ojanguren, "Modelización global del proceso de colada continua", Revista de Metalurgia., Vol. Extr. pp. 463-468, 2005. https://doi.org/10.3989/revmetalm.2005.v41.iExtra.1077

[43] J. L. Acevedo Cabello, "Predicción microestructural de palanquillas de acero al carbono obtenidas por colada continua empleando una aproximación macro-micro", (Tesis Doctoral), Universitat Politécnica de Catalunya, España, 2013. URL

[44] M. Shen; Z. Zang; K. Shu, "Mathematics simulation and experiments of continuous casting with strip feeding in mold”. Metalurgija, vol. 56, no. 3-4, pp. 315-318, 2017. URL

[45] R. Alberny; A. Leclercq; D. Aumary; M. Lahousse, "La lingotière de coulée continue de brames et son bilan thermique", Revista Metalúrgica., vol. 73, no. 7-8, pp. 545-558. Jul. 1976. https://doi.org/10.1051/metal/197673070545

[46] E. A. Mizikar, "Mathematical heat transfer model for solidification of continuously cast steel slabs", The American Institute of Mining, Metallurgical, and Petroleum Engineers, PART XI - November.1968. URL

[47] Z. Peng; Y. ping Bao; Y. nan Chen; L. kang Yang; C. Xie; F. Zhang, "Effects of calculation approaches for thermal conductivity on the simulation accuracy of billet continuous casting"., Int. J. Miner. Metall. Mater., vol. 21, 18-25. Jan. 2014. https://doi.org/10.1007/s12613-014-0860-6

[48] B.G. Thomas; I. V. Samarasekera; J. K. Brimacombe, "Comparison of numerical modeling techniques for complex, two-dimensional, transient heat-conduction problems"., Metall. Mater. Trans B., vol. 15, pp. 307318, Aug. 1984. https://doi.org/10.1007/BF02667334

[49] J. Szekely; V. Stanek, "On heat transfer and liquid mixing in the continuous casting of steel"., Metall. Trans., vol. 1, pp. 119-126. Apr. 1970. URL

[50] A. Ramírez-López; R. Aguilar-López; M. Palomar-Pardavé; M. A. Romero; D. Muñoz-Negrón, "Simulation of heat transfer in steel billets during continuous casting”., Int. J. Miner. Metall. Mater. vol. 17, pp. 403416. Jul. 2010. https://doi.org/10.1007/s12613-010-0333-5

[51] M. Vynnycky, "Applied mathematical modelling of continuous casting processes: A Review", Metals, vol. 8, no. 11, nov. 2018. https://doi.org/10.3390/met8110928

[52] J. Madias, "Innovaciones en la colada continua de semiproductos para laminados largos", Acero Latinoamericano., no. 567, pp. 26- 37, Mar, 2018. URL

[53] I. V. Samarasekera; D. L. Anderson; J. K. Brimacombe, "The Thermal Distortion of Continuous-Casting Billet Molds", Metallurgical Transactions B., vol. 13B, pp. 91-104. Dec. 1980. https://doi.org/10.1007/BF02666960

[54] I. V. Samarasekera; J. K. Brimacombe, "The Influence of Mold Behavior on the Production of Continuously Cast Steel Billets", Metallurgical Transactions B. vol. 13, pp. 105-116. Dec. 1982. https://doi.org/10.1007/BF02666961

[55] P. Ni; M. Ersson; L. Ingemar Jonsson; T. Zhang; P. Jönsson, "Numerical Study on the Influence of a Swirling Flow Tundish on Multiphase Flow and Heat Transfer in Mold”, Metals, vol. 8, no. 5, pp. 368, May. 2018. https://doi.org/10.3390/met8050368

[56] R. Manojlovic, "Mathematical modeling of solidification Process of continuous casting steel slabs", Journal of Chemical Technology and Metallurgy, vol. 48, no. 4, pp. 419-427, May. 2013. URL

[57] B. Thomas; L. Zhang, "Mathematical Modeling of Fluid Flow in Continuous Casting", ISIJ International, vol. 41, no. 10, pp. 1181-1193, Oct. 2001. https://doi.org/10.2355/isijinternational.41.1181

[58] H. T. Abuluwefa; M. A. Al-Ahresh; A. Bosen, "Factors Affecting Solidification of Steel in the Mould During Continuous Casting of Steel Billets", Procedures of the international multiconference of engineers and computer scientists, Hong Kong, Vol. II, 2012, pp. 14-16. URL

[59] L. Bai; B. Wang; H. Zhong; J. Ni; Q. Zhai; J. Zhang, "Experimental and numerical simulations of the solidification process in continuous casting of slab", Metals, vol. 6, no. 3, Mar. 2016. https://doi.org/10.3390/met6030053

[60] E. I. Peterson, "Mold flux crystallization and mold thermal behavior", (Tesis de Maestría), Missouri University of Science and Technology, 2017. URL

[61] Y. Kong; D. Chen; Q. Liu; M. Long, "A Prediction Model for Internal Cracks during Slab Continuous Casting”, Metals, vol. 9, no. 5, pp. 587, May. 2019. https://doi.org/10.3390/met9050587 
[62] K. Tsutsumi; T. Nagasaka; M. Hino, "Surface roughness of solidified mold flux in continuous casting process”, ISIJ international, vol. 39, no. $11, \quad$ pp. $1150-1159 . \quad 1999$. https://doi.org/10.2355/isijinternational.39.1150

[63] G. Krauss, "Solidification, segregation, and banding in carbon and alloy steels", Metall Mater Trans B, vol. 34, no. 6, pp. 781-792, Dec. 2003. https://doi.org/10.1007/s11663-003-0084-Z

[64] A. Bermúdez, "Modelos matemáticos en solidificación, Aplicaciones en metalurgia", Pub. Mat. UAB. no. 22, pp. 213-222, 1980. https://doi.org/10.5565/publmat_22180_43

[65] A. Grill; J. K. Brimacombe, "Influence of carbon content on rate of heat extraction in the mould of a continous-casting machine", Ironmaking Steelmaking, vol. 3, no. 2, pp. 76-79, 1976. URL

[66] H. Cui; K. Zhang; Z. Wang; B. Chen; B. Liu; J. Qing; Z. Li, "Formation of Surface Depression during Continuous Casting of High-Al TRIP Steel”. Metals, vol. 9, no. 2, pp. 204, Feb. 2019. https://doi.org/10.3390/met9020204

[67] J. Saavedra Poma, "Influencia de la transferencia de calor en la formación de grietas internas". (Tesis pregrado), Universidad Nacional de Ingeniería, Facultad de Ingeniería Geológica, Minera y Metalúrgica. Lima, Perú, 2009. URL

[68] I. V. Samarasekera, "Thermal distortion of continuous casting moulds". (Tesis Doctoral) The University of British Columbia, Vancouver, Canada, 1980. URL

[69] J. Elfsberg, "Oscillation Mark Formation in Continuous Casting Processes". (Tesis pregrado), Royal Institute of Technology SE-100 44 Stockholm, Sweden, 2003. URL

[70] X. Sun; B. Li; H. Lu; Y. Zhong; Z. Ren; Z. Lei, "Steel/Slag Interface Behavior under Multifunction Electromagnetic Driving in a Continuous Casting Slab Mold”, Metals, vol. 9, no. 9, 983, Sep. 2019. https://doi.org/10.3390/met9090983

[71] E. Brandaleze; E. González; M. Bentancour, "Mediciones del Porcentaje de Cristalinidad y Determinación del Comportamiento de Polvos Coladores en el Rango entre $1000{ }^{\circ} \mathrm{C}$ Y $1200{ }^{\circ} \mathrm{C}$ ", Revista Materia, vol. 8, no. 3, pp. 238 - 248. 2003. URL

[72] A. Sorek; Z. Kudlinski, "The influence of the near-meniscus zone in continuous casting mold on the surface quality of the continuous casting ingots". Archives of Metallurgy and Materials., vol. 57, no. 12012. https://doi.org/10.2478/v10172-012-0036-1

[73] R. Chaudhary; G. Lee; B. G. Thomas; S. Cho; S. H. Kim; O. D. Kwon. "Effect of Stopper-Rod Misalignment on Fluid Flow in Continuous Casting of Steel". Metallurgical and Materials Transactions B, vol. 42, no. 2, pp. 300-315, Feb. 2011. https://doi.org/10.1007/s11663-011-9478-5

[74] A. Robles Álvarez; H. López García; P. Fernández-Cueto Arguedas; A. M. Díaz Fernández; L. F. Sancho Méndez "Predicción de CLOGGING en la colada continua mediante análisis del sistema de control de nivel", In XXIX Jornadas de Automática, Tarragona, 2008. URL

[75] D. Wu; S. Cheng; J. Zhao, "Performance comparison of three kinds of submerged entry nozzles for bloom mold", J. Iron Steel Res. Int., vol. 15, pp. 315-321, 2008. URL

[76] M. Long; H. Chen; D. Chen; S. Yu; B. Liang; H. Duan, "A Combined Hybrid 3-D/2-D Model for Flow and Solidification Prediction during Slab Continuous Casting”, Metals, vol. 8, no. 3, Mar. 2018. https://doi.org/10.3390/met8030182

[77] H. Sun; L. Li; C. Liu, "Novel Opposite Stirring Mode in Bloom Continuous Casting Mould by Combining Swirling Flow Nozzle with EMS”, Metals, vol. 8, no. 10, pp. 842, 2018. https://doi.org/10.3390/met8100842

[78] H. Sun; J. Zhang, "Macrosegregation improvement by swirling flow nozzle for bloom continuous castings", Metall. Mater. Trans. B, vol. 45, pp. 936-946, Dec. 2014. https://doi.org/10.1007/s11663-013-9999-1

[79] P. Wang et al., "Initial Transfer Behavior and Solidification Structure Evolution in a Large Continuously Cast Bloom with a Combination of Nozzle Injection Mode and M-EMS”, Metals, vol. 9, no. 10, pp. 1083, Oct. 2019. https://doi.org/10.3390/met9101083

[80] C. Real; L. Hoyos; F. Cervantes; R. Miranda; M. Palomar; J. González, "Dinámica de fluidos en una Buza Bifurcada y su influencia en un molde de colada continua”, $8^{\circ}$ Congreso Iberoamericano de Ingeniería Mecánica, Cusco, 2007. URL

[81] K. Rackers; B. Thomas, "Clogging in Continuous Casting Nozzles", 78th Steelmaking Conference Proceedings, Nashville, TN, 1995. URL

[82] M. H. Zare; A. H. Meysami; S. Mahmoudi; M. Hajisafari; M. MazarAtabaki. "Simulation of fluid flow and solidification in the funnel type crystalizer of thin slab continuous cast". Orient $J$ Chem, vol. 29, no. 4, Jan. 2014. URL

[83] D. Jiang; M. Zhu; L. Zhang, "Numerical Simulation of Solidification Behavior and Solute Transport in Slab Continuous Casting with S-EMS”, Metals, vol. 9, no. 4, pp. 452, Apr. 2019. https://doi.org/10.3390/met9040452

[84] M. Guimarães; A. L. Vasconcellos da Costa e Silva, "Evaluating segregation in HSLA steels using computational thermodynamics", Journal of Materials Research and Technology, vol. 4, no. 4, pp. 353-358, Dec. 2015. https://doi.org/10.1016/j.jmrt.2015.06.002 
[85] W. Zhang; S. Luo; Y. Chen; W. Wang; M. Zhu. "Numerical Simulation of Fluid Flow, Heat Transfer, Species Transfer, and Solidification in Billet Continuous Casting Mold with M-EMS". Metals, vol. 9, no. 1, pp. 66. Jan. 2019. https://doi.org/10.3390/met9010066

[86] M. C. Flemings, "Solute Segregation", Encyclopedia of Materials: Science and Technology (Second Edition). pp. 8753-8755, 2001. https://doi.org/10.1016/B0-08-043152-6/01566-7

[87] P. Emtage; K. Wunnenberg; T. Hatonen; M. Bobadilla; J. Llanos; M. De Santis, "Improved Control of Segregation in Continuous Casting and Hot Rolling Processes", European Commission, Luxembourg, International, reporte EUR 20886 En, pp. 1-403, 2003. URL

[88] R. Niu; B. Li; Z. Liu; X. Li, "Melting of Moving Strip during Steel Strip Feeding in Continuous Casting Process", Steel research international, vol. 89, no. 5, 2-13. Feb. 2018. https://doi.org/10.1002/srin.201700407

[89] M. Vynnycky; S. Saleem; K. M. Devine; B. J. Florio; S. L. Mitchell; S. B. G. O’Brien, "On the formation of fold-type oscillation marks in the continuous casting of steel”, $R$. Soc. open sci. vol. 4, no. 6. Jun. 2017. https://doi.org/10.1098/rsos.170062

[90] R. López; J. Usart; D. Cerutti, "Medición de nivel en los moldes de colada continua". $46^{\circ}$ Seminario de Aceria Internacional. ABM Week, Rio de Janeiro, 2015. URL

[91] X. Yan; B. Jia; Q. Wang; S. He; Q. Wang, "Mold nonsinusoidal oscillation mode and its effect on slag infiltration for lubrication and initial shell growth during steel continuous casting", Metals, vol. 9, no. 4, pp. 418, Apr. 2019. https://doi.org/10.3390/met9040418

[92] H. Wu; Y. Xu; Z. Huo; F. Yue; P. Lu, "Physical modeling of oscillation Effect on fluid flow in mold", Metalurgija vol. 54, no. 3, pp. 465-468, 2015. URL

[93] J. Sengupta; B. G. Thomas, "Visualization of Hook and Oscillation Mark Formation Mechanism in UltraLow Carbon Steel Slabs During Continuous Casting”. JOMe, Journal of Metals - electronic edition, pp. 121, Dec. 2006. URL

[94] J. Cibulka; R. Krzok; R. Hermann; D. Bocek; J. Cupek; K. Michalek, "Impact of oscillation parameters on surface quality of cast billets", Arch. Metall. Mater. vol. 61, no 1, pp. 283-288, 2016. https://doi.org/10.1515/amm-2016-0054

[95] O. Pütz; O. Breitfeld; S. Rödl. "Investigations of Flow Conditions and Solidification in Continuous Casting Moulds by Advanced Simulation Techniques". Steel Research International, vol. 74, no.11-12, pp.686-692. 2003. URL

[96] Q. Fang; H. Ni; B. Wang; H. Zhang; F. Ye, "Effects of EMS Induced Flow on Solidification and Solute Transport in Bloom Mold", Metals, vol. 7, no. 3, pp. 72, Feb. 2017. https://doi.org/10.3390/met7030072

[97] L. Hui-cheng; L. Yu-xiang; Z. Yun-hu; L. Zhen; Z. Qi-Jie Zhai, "Effects of hot top pulsed magneto-oscillation on solidification structure of steel ingot", China Foundry, vol. 15, no. 2, pp. 110-116, Mar, 2018. https://doi.org/10.1007/s41230-018-7198-Z

[98] B. Wang; Z. Yang, X. Zhang, Y. Wang, C. Nie, Q. Liu; H. Dong, "Analysis of the effects of electromagnetic stirring on solidification structure of bearing steel”, Metalurgija, vol. 54, no. 2, pp. 327-330, 2015. URL 